\title{
PTF14jg: The Remarkable Outburst and Post-burst Evolution of a Previously Anonymous Galactic Star
}

\author{
Lynne A. Hillenbrand ${ }^{1}$, Adam A. Miller ${ }^{1,2}$ (10), John M. Carpenter ${ }^{1,3}$ (D), Mansi M. Kasliwal ${ }^{1}$ (i), Howard Isaacson $^{4}$ (1), \\ Sumin Tang ${ }^{1,5}\left(10\right.$, Vishal Joshi ${ }^{6}$ (i) D. P. K. Banerjee ${ }^{6}$, and Roc M. Cutri ${ }^{7}$ (i) \\ ${ }^{1}$ Department of Astronomy, California Institute of Technology, Pasadena, CA 91125, USA \\ ${ }^{2}$ Center for Interdisciplinary Exploration and Research in Astrophysics (CIERA), Northwestern University, 2145 Sheridan Road, Evanston, IL 60208, USA \\ ${ }^{3}$ Joint ALMA Observatory, Avenida Alonso de Cordova 3107, Vitacura, Santiago, Chile \\ ${ }^{4}$ University of California at Berkeley, Berkeley, CA, USA \\ ${ }^{5}$ Kavli Institute for Theoretical Physics, University of California, Santa Barbara, CA 93106, USA \\ ${ }^{6}$ Astronomy and Astrophysics Division, Physical Research Laboratory, Navrangpura, Ahmedabad, Gujarat 380 009, India \\ ${ }^{7}$ Infrared Processing and Analysis Center, California Institute of Technology, Pasadena, CA 91125, USA \\ Received 2018 July 27; revised 2019 January 27; accepted 2019 January 28; published 2019 March 26
}

\begin{abstract}
We report the outbursting source PTF 14jg, which, prior to the onset of its late 2013 eruption, was a faint, unstudied, and virtually uncataloged star. The salient features of the PTF 14jg outburst are (i) projected location near the W4 H II region and radial velocity consistent with physical association; (ii) a light curve that underwent an $\sim 6-7$ mag optical ( $R$-band) through mid-infrared ( $L$-band) brightening on a timescale of a few months, peaked and then faded by $\sim 3 \mathrm{mag}$, but plateaued still $>3.5 \mathrm{mag}$ above quiescence by $\sim 8$ months post-peak, lasting to at least $4 \mathrm{yr}$ after eruption; (iii) strong outflow signatures with velocities reaching $-530 \mathrm{~km} \mathrm{~s}^{-1}$; (iv) a low-gravity and broad ( 100-150 $\mathrm{km} \mathrm{s}^{-1}$ FWHM) optical absorption-line spectrum that systematically changes its spectral type with wavelength; (v) lithium; and (vi) ultraviolet and infrared excess. We tentatively identify the outburst as exhibiting characteristics of a young star FU Ori event. However, the burst would be unusually hot, with an absorption spectrum exhibiting high-excitation $(\sim 11,000-15,000 \mathrm{~K})$ lines in the optical and no evidence of $\mathrm{CO}$ in the near-infrared, in addition to exhibiting an unusual light curve. We thus also consider alternative scenariosincluding various forms of novae, nuclear-burning instabilities, massive star events, and mergers-finding them all inferior to the atypically hot FU Ori star classification. The source eventually may be interpreted as a new category of young star outburst with a larger amplitude and shorter rise time than most FU Ori-like events.
\end{abstract}

Key words: circumstellar matter - infrared: stars - stars: activity - stars: pre-main sequence - stars: variables: general - stars: winds, outflows

Supporting material: data behind figures, machine-readable tables

\section{Introduction}

The $W 3 / W 4 / W 5$ complex in the Perseus spiral arm is one of the more dynamic regions of ongoing star formation in the Galaxy (see review by Megeath et al. 2008 and references therein). It is large and massive, spanning $\sim 200 \times 50 \mathrm{pc}^{2}$ with several times $10^{5} M_{\odot}$ in molecular gas. In addition to several rich star clusters containing tens of thousands of stars, it features ionized gas localized in $\mathrm{H}$ II regions and neutral gas that has been shaped into shells and chimneys. Further evidence of the interaction of massive stars with the local interstellar medium includes numerous supernova remnants, large wind-blown bubbles, cometary and fragmented clouds, and sculpted dust pillars.

The object of interest identified here, PTF $14 \mathrm{jg}$, is located at 02:40:30.14 + 60:52:45.5 $(l=135.81524, b=0.76533)$. In the plane of the sky, the position is between two of the sequence of three large H II regions, just east of the "swept-up shell" in W4 (see the H I and dust maps in Terebey et al. 2003, their Figures 2 and 3 ) and $\sim 1.1$ from the center of the massive cluster IC 1805. The position does not appear to be associated with significant molecular gas emission (e.g., Carpenter et al. 2000, their Figures 1 and 2; Lagrois \& Joncas 2009, their Figure 9) or any of the identified infrared clusters. Many surveys targeting the stellar population of the $\mathrm{H}$ II regions have missed covering this area. Thus, there is no SIMBAD counterpart to PTF $14 \mathrm{jg}$, meaning there is no previous literature. There is also no corresponding object in Vizier, meaning there is no documented photometric detection at any wavelength. The evidence below, primarily radial velocity information, associates PTF 14jg with the Perseus arm. Based on parallaxes of masing sources in W3, Xu et al. (2006) derived a distance of $1.95 \mathrm{kpc}$, which is commonly adopted as the distance to the overall complex.

In this paper, we present the substantial optical brightening of PTF 14jg that occurred over a time period of several months in 2013-2014 and our multiwavelength photometric and spectroscopic follow-up observations during the subsequent several years. We draw analogies between this source and the rarely populated FU Ori class (Herbig 1977; Hartmann \& Kenyon 1996) of young stellar objects.

The FU Ori stars are interpreted as young low-mass stars undergoing episodes of rapid accretion at rates that are three to four orders of magnitude larger than the quiescent-state $\mathrm{T}$ Tauri accretion rates, typically $\sim 10^{-8} M_{\odot} \mathrm{yr}^{-1}$ (e.g., Gullbring et al. 1998). The occurrence rate and duty cycle of FU Ori events is crucial to establish, given our poor understanding of accretion histories during star formation and pre-main-sequence evolution. However, the known FU Ori population is small. The class is an empirically diverse set of objects that are associated together based mainly on the definitive spectral type change with wavelength, strong outflow signatures, infrared excess, and an observed or suspected large-amplitude, long-duration 

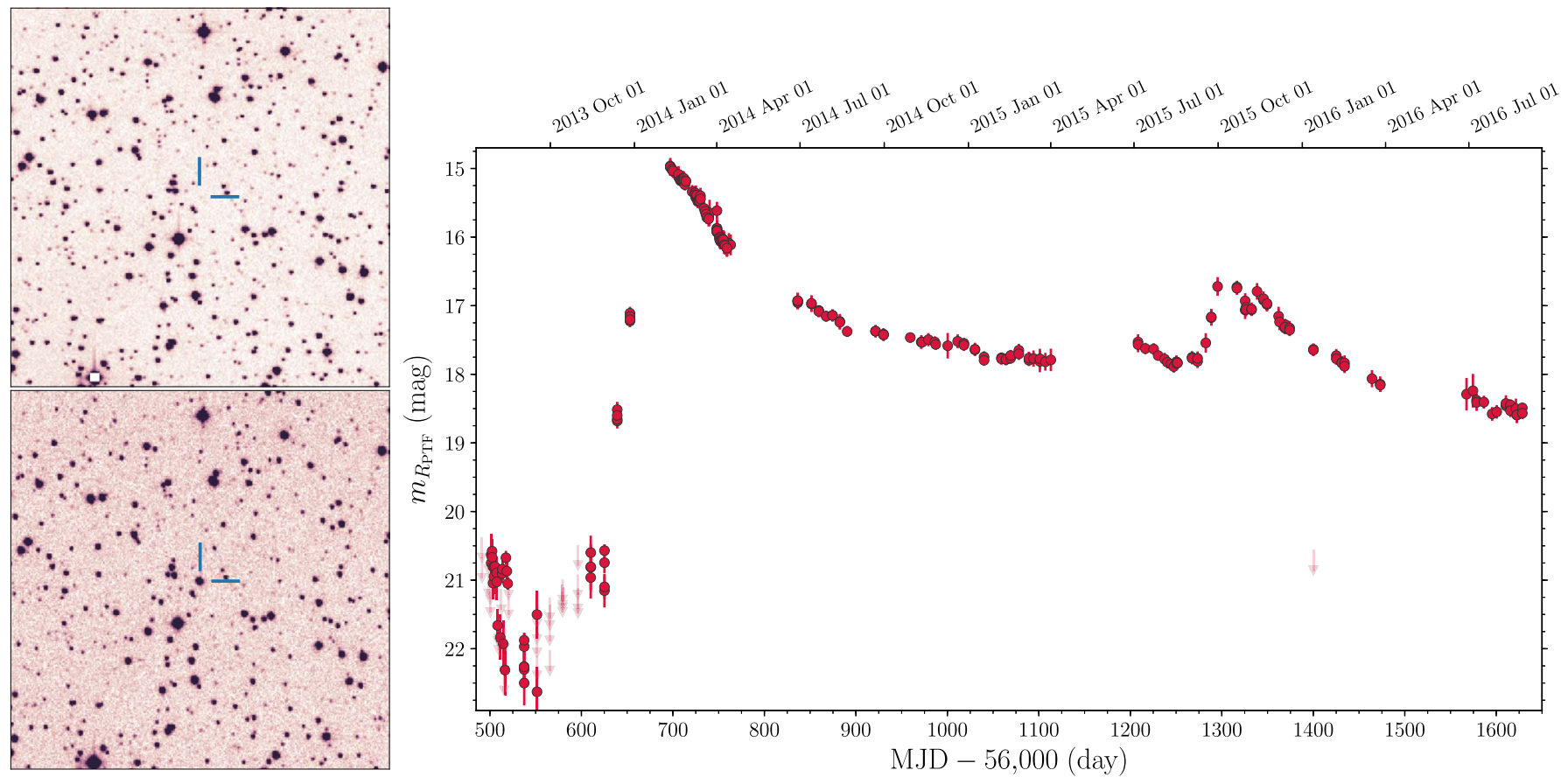

Figure 1. Left: PTF image over $5^{\prime} \times 5^{\prime}$ in 2013 before the outburst (top panel), created from an image stack of available data, and on 2014 February 9 , near the peak of the light curve (bottom panel). Right: $R_{\mathrm{PTF}}$ light curve beginning in late 2013 and extending through late 2016. There is evidence for a secondary maximum occurring when the object was behind the Sun in mid-2015 and detection of a tertiary maximum during late 2015.

photometric outburst (Reipurth \& Aspin 2010). Furthermore, relatively few FU Ori stars were observed as their outbursts occurred, and even fewer were well studied before their outbursts. Thus, our knowledge of the progenitors, the range in light-curve rise shapes, and the early outburst spectroscopic characteristics is severely limited.

Section 2 reports our detection of PTF 14jg from quiescence to outburst, and Section 3 discusses archival information at the outburst position. Section 4 presents the follow-up photometry and spectroscopy we collected, Section 5 is our analysis of the outburst colors and spectral energy distribution (SED), Section 6 is our analysis of the multiyear light curve, and Section 7 is our analysis of the complex picture presented in the spectroscopy. A synthesis of the observational evidence in the context of the FU Ori interpretation is presented in Section 8, while Section 9 considers other possible interpretations of the collected evidence regarding PTF 14jg. Section 10 contains our discussion, and Section 11 presents a short conclusion.

\section{Detection of the PTF 14jg Outburst Event}

The (intermediate) Palomar Transient Factory (Law et al. 2009; Kulkarni 2013) monitored the $W 3 / W 4 / W 5$ complex in the $R$ band beginning in the summer of 2013. After initial experimentation with the viability of this crowded field in Cassiopeia for automated processing and photometry, lowcadence (once every 2 weeks) observations began in the fall of 2013. A new source was identified as a candidate transient on 2014 January 24 and given the name PTF 14jg. We estimate that PTF 14jg achieved peak brightness around 2014 February 9, then decreased in brightness (see Figures 1 and 2).

Faint photometry recovered (Laher et al. 2014) from observations taken before the outburst shows that PTF 14jg exhibited significant variability during 2013 July through September at about the $1 \mathrm{mag}$ level, in the range
$R_{\mathrm{PTF}}=21-22^{m}$, including several coherent dips in brightness on a timescale of about 5 days. In 2013 December, the source began to gradually increase its brightness from $R_{\mathrm{PTF}}<21^{m}$ to $R_{\mathrm{PTF}} \approx 15^{m}$ by 2014 mid-February, i.e., a $>6$ mag rise in $\sim 70$ days. The light curve was subsequently sampled with nightly cadence but interrupted due to the prescribed PTF switch from $R$-band observing to $\mathrm{H} \alpha$-filter observing on the 5 days around full moon. The realized cadence was further subject to some poor weather and continuing monthly gaps around new moon, as well as a longer gap when the object set for the 2013-2014 season. Nevertheless, the light curve has good coverage of its main features. The cadence was decreased for the 2014-2015 season and those beyond.

The photometry reported in Table 1 was obtained with the Palomar 48 inch telescope (P48) and the PTF Survey Camera as part of routine iPTF operations. The $R_{\mathrm{PTF}}$ band is approximately a Mould $R$ filter (Law et al. 2009). Observations are calibrated relative to Sloan Digital Sky Survey (SDSS; York et al. 2000) stars, which are observed throughout the night (see Ofek et al. 2012 for further details). Photometry of PTF 14jg was measured using the custom point-spread function fitting routine PTFIDE (Masci et al. 2017).

\section{Archival Information}

A search of available image and catalog data from optical survey sources (e.g., DSS, iPHAS, UVEX) does not reveal a previous detection at the position of PTF 14jg. Pan-STARRS appears to have caught the burst, though individual-epoch measurements that potentially include pre-burst as well as inburst magnitudes are not yet available in the DR1 catalog. The source is not in the near-infrared 2MASS catalog (Cutri et al. 2003). The most sensitive near-infrared catalog, UKIDSS, missed covering this high-declination field. In the mid-infrared, 

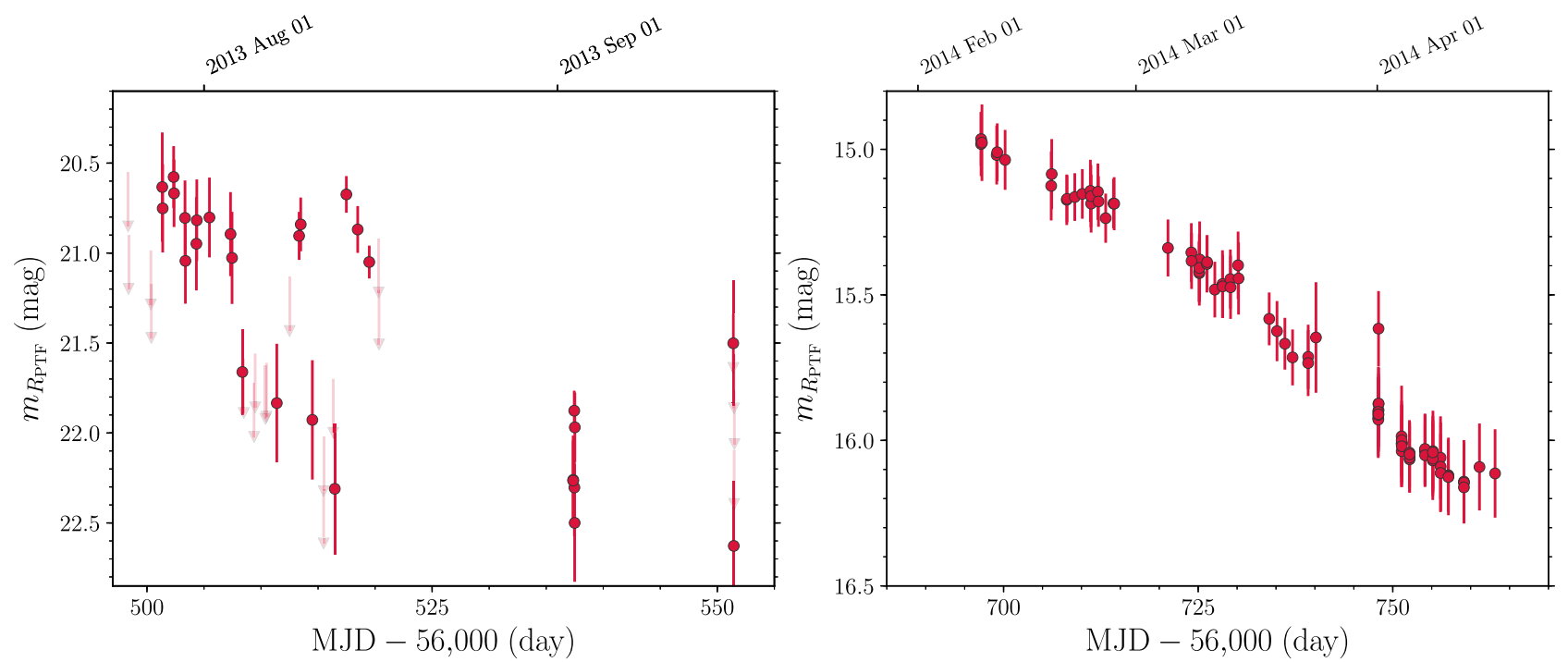

Figure 2. Expanded view of the Figure 1 light curve, highlighting the pre-outburst brightness variations in late 2013 (left panel) and the initial decline from the absolute light-curve peak in early 2014 (right panel), which appears to change slope around day 730. Note that the ordinate scaling is different between the two panels.

Table 1

P48 R-band Observations

\begin{tabular}{lcc}
\hline \hline MJD & $\begin{array}{l}m_{R \text { PTF }} \\
(\mathrm{mag})\end{array}$ & $\begin{array}{c}\sigma_{\mathrm{m}} \\
(\mathrm{mag})\end{array}$ \\
\hline 56491.369 & 20.38 & $\ldots \ldots^{\mathrm{a}}$ \\
56491.405 & 20.67 & $\ldots{ }^{\mathrm{a}}$ \\
56498.349 & 20.55 & $\ldots{ }^{\mathrm{a}}$ \\
56498.386 & 20.90 & $\ldots{ }^{\mathrm{a}}$ \\
56500.345 & 20.99 & $\ldots{ }^{\mathrm{a}}$ \\
56500.383 & 21.17 & 0.30 \\
56501.343 & 20.63 & 0.25 \\
56501.380 & 20.75 & 0.17 \\
56502.339 & 20.58 & 0.19 \\
56502.375 & 20.67 & \\
\hline
\end{tabular}

Note.

${ }^{\mathrm{a}} 4 \sigma$ statistical upper limit.

(This table is available in its entirety in machine-readable form.)

PTF 14jg was not detected in the WISE all-sky survey (Cutri et al. 2012), which had lower spatial resolution and sensitivity relative to Spitzer.

The source was observed as part of the GLIMPSE-360 survey with Spitzer (Hora et al. 2007; Churchwell et al. 2009). The data products available at NASA's IRSA ${ }^{8}$ show a detection within 0".44 in the GLIMPSE-360 Catalog, but only in the $3.6 \mu \mathrm{m}$ band at $17.2 \pm 0.1 \mathrm{mag}$. However, the "more complete, less reliable" GLIMPSE-360 Archive reports a [3.6] = $17.21 \pm 0.12$ and $[4.5]=16.82 \pm 0.18$ source. The infrared color is therefore [3.6]-[4.5] $=0.39 \pm 0.22$. For the average pre-outburst optical brightness of $R_{\mathrm{PTF}}=21.5$, the opticalinfrared color $R_{\mathrm{PTF}}$ [3.6] is therefore $4.3 \mathrm{mag}$.

Although red, the source would not have been identified as a clear candidate young star based on its infrared color alone. At these faint magnitudes, a color of [3.6]-[4.5] $>0.5$ would be required in order to be distinguishable from noise among the field star population for a source that is not also detected at longer infrared wavelengths.

\footnotetext{
8 https://irsa.ipac.caltech.edu/data/SPITZER/GLIMPSE/overview.html
}

Photosphere models for stars predict essentially zero [3.6][4.5] color and increasing to a maximum color of only $0.15 \mathrm{mag}$ for low-mass brown dwarfs. For PTF 14jg, a color excess of $0.1-0.4$ mag suggests the presence of circumstellar dust in the pre-outburst stage. A dust excess is also revealed in our more considered SED analysis below (Section 5).

\section{Photometric and Spectroscopic Follow-up Observations}

Our follow-up to the dramatic optical brightening event in PTF 14jg includes photometric and spectroscopic observations, in addition to continued Palomar 48 inch $R$-band monitoring. Optical and near-infrared photometric data were obtained at the Palomar 60 inch telescope (ugriz) and Mount Abu $1.2 \mathrm{~m}$ telescope $\left(J H K_{s}\right)$, while optical and infrared spectra were obtained at the Apache Point Observatory, Palomar Observatory, and Keck Observatory. We also acquired bluerwavelength ultraviolet and X-ray data from UVOT on board $S w i f t^{9}$ and redder-wavelength mid-infrared (Spitzer, NEOWISE) and millimeter (CARMA) observations.

Details of the follow-up observations appear in the tables and are described below. All follow-up observations occurred after the light-curve peak in early February of 2014.

\subsection{X-Ray and UV Photometry}

Space-based ultraviolet and optical observations were obtained with the UVOT instrument (Roming et al. 2005). On 2014 March 28, April 6, and June 25, PTF14jg was observed in all six UVOT filters. Photometry was performed and calibrated using standard UVOT tools (see Breeveld et al. 2010 ) and is reported in Table 2 on the AB mag system. In the $\mathrm{X}$-ray channel, only an upper limit of 0.00235 counts $\mathrm{s}^{-1}$ was derived from $6414 \mathrm{~s}$ of integration.

\subsection{Optical Photometry}

Observations at the Palomar 60 inch telescope were conducted using the GRB camera (Cenko et al. 2006) in the ugriz bands starting on 2014 February 9 UT. Aperture

\footnotetext{
9 In early 2018, Swift was renamed the Neil Gehrels Swift Observatory.
} 
Table 2

Swift UVOT Observations

\begin{tabular}{|c|c|c|c|c|c|c|}
\hline Filter & $\begin{array}{c}t_{\text {start }} \\
\text { (MJD) }\end{array}$ & $\begin{array}{c}t_{\text {end }} \\
(\mathrm{MJD})\end{array}$ & $\begin{array}{c}\text { Exp. } t \\
\text { (s) }\end{array}$ & $\begin{array}{l}\mathrm{mag} \\
(\mathrm{AB})\end{array}$ & $\begin{array}{c}\sigma_{\text {mag }} \text { stat. } \\
\text { (AB) }\end{array}$ & $\begin{array}{c}\sigma_{\mathrm{mag}} \text { sys } \\
\text { (AB) }\end{array}$ \\
\hline$\overline{\text { UVW2 }}$ & 56744.04 & 56744.24 & 498.0 & $>22.07^{\mathrm{a}}$ & $\ldots$ & $\ldots$ \\
\hline UVM2 & 56744.04 & 56744.24 & 935.8 & $>22.28^{\mathrm{a}}$ & $\ldots$ & $\ldots$ \\
\hline$U$ & 56744.04 & 56744.24 & 99.5 & 18.62 & 0.13 & 0.02 \\
\hline$B$ & 56744.04 & 56744.24 & 99.5 & 17.33 & 0.09 & 0.02 \\
\hline V & 56744.04 & 56744.24 & 99.5 & 16.59 & 0.09 & 0.01 \\
\hline UVW1 & 56753.63 & 56753.70 & 312.5 & 21.41 & 0.36 & 0.03 \\
\hline$U$ & 56753.63 & 56753.70 & 103.9 & 19.23 & 0.17 & 0.02 \\
\hline$B$ & 56753.63 & 56753.70 & 103.9 & 17.70 & 0.10 & 0.02 \\
\hline V & 56753.64 & 56753.70 & 103.9 & 16.72 & 0.09 & 0.01 \\
\hline UVW2 & 56833.55 & 56833.62 & 555.7 & $>22.26^{\mathrm{a}}$ & $\ldots$ & $\ldots$ \\
\hline UVM2 & 56833.55 & 56833.62 & 834.8 & $>22.25^{\mathrm{a}}$ & $\ldots$ & $\ldots$ \\
\hline
\end{tabular}

Note.

a $3 \sigma$ statistical upper limit.

photometry was performed using SExtractor (Bertin \& Arnouts 1996) and is reported in Table 3.

The griz data were calibrated using six secondary standards, whose brightness was determined relative to SDSS stars observed by P60 on 2014 February 11 UT, a photometric night. Based on the scatter in the photometric solution, there is an $\sim 0.04$ mag systematic uncertainty for the reported griz photometry. There was an insufficient number of P60 $u$-band observations of SDSS fields to determine secondary $u$-band standards; thus, the P60 $u$ observations were calibrated using the Swift/UVOT $U$-band observations of the field. In the two epochs of UVOT observations, there were 10 bright ( $U<18 \mathrm{mag}$ ) stars that did not show significant variations, which we adopt as our $U$-band secondary standards. The UVOT $u$ filter does not perfectly match the one employed by the P60; thus, we expect an $\sim 0.1$ mag systematic uncertainty associated with these calibrations.

Figure 3 shows the multiwavelength evolution in brightness, and Figure 4 shows the color evolution. No colors are available during the outburst, but the decline from peak brightness shows little color change at optical wavelengths until after about one $e$-folding time, when the source appears to become slightly redder by about $0.2 \mathrm{mag}$ in $g-r$ and $0.1 \mathrm{mag}$ in $r-i$. Adopting the source extinction estimated below of $A_{V}=$ $4.75 \mathrm{mag}$ and dereddening the colors at the outburst peak results in intrinsic $u-g \sim 0.0, g-r \sim-0.7, r-i \sim-0.4$, and $i-z \sim-0.5$.

\subsection{Near-infrared Photometry}

Near-infrared photometric observations were carried out from the $1.2 \mathrm{~m}$ telescope of the Mount $\mathrm{Abu}$ Infrared Observatory using the Near-Infrared Camera/Spectrometer (NICS) equipped with a $1024 \times 1024 \mathrm{HgCdTe}$ Hawaii 1 array (Anandarao et al. 2008; Banerjee \& Ashok 2012). The camera has an unvignetted $8 \times 8 \operatorname{arcmin}^{2}$ field and uses $J H K_{s}$ filters that conform to Maunakea Observatories (MKO) specifications. Frames in each filter were obtained in five dithered positions, typically offset by $30^{\prime \prime}$, with multiple frames (generally five) being obtained in each dithered position. Dark frames of the same integration time as those used for the science frames were acquired.

Bad-pixel masking was applied, and corrections were made for cosmic-ray hits. The dark-subtracted dithered frames were median-combined to produce a sky-flat frame. Individual frames were then divided with the sky flat normalized to unity. The flat-corrected science frames were again mediancombined in each filter to produce a median sky frame, which was subtracted from the individual science frames in order to correct for bias, dark, and sky background. The flat-fielding and sky subtraction methods adopted are customary techniques in the near-infrared. ${ }^{10}$ Finally, the corrected science frames were coadded to produce an average frame on which photometry was done.

Aperture photometry was derived using the aperture photometry routines in IRAF, with the nearby stars 2MASS $\mathrm{J} 02403063+6051272$ and $\mathrm{J} 02403914+6052201$ (avoiding the nearest bright star to PTF 14jg, which appears to be a variable) used for photometric calibration. Because NICS employs MKO filters, the 2MASS magnitudes of the calibrating stars were first converted to the MKO near-infrared photometric system using the transformation equations of Leggett et al. (2006). The resulting $\mathrm{JHK}_{s}$ magnitudes of PTF $14 \mathrm{jg}$ in the MKO photometric system are listed in Table 4, and the photometry is illustrated in Figure 5.

The infrared burst colors are redder than can be explained by reddened stellar photospheres and consistent with the colors of young star-plus-circumstellar dust systems. Unlike in the optical, following its peak brightness, PTF 14jg exhibited a color change in the near-infrared during its early decline (Figure 4), becoming redder. If interpreted as increasing extinction, the change corresponds to almost 0.4 mag in $A_{K}$

\footnotetext{
${ }_{10}$ www.cfht.hawaii.edu/Instruments/Detectors/IR/Redeye/Manual/ chapter7.html
} 
Table 3

P60 ugriz Observations

\begin{tabular}{|c|c|c|c|c|c|c|c|c|c|c|c|c|c|c|}
\hline $\mathrm{MJD}_{u}$ & $\begin{array}{c}u \\
(\mathrm{mag})\end{array}$ & $\begin{array}{c}\sigma_{u} \\
(\mathrm{mag})\end{array}$ & $\mathrm{MJD}_{g}$ & $\begin{array}{c}g \\
(\mathrm{mag})\end{array}$ & $\begin{array}{c}\sigma_{g} \\
(\mathrm{mag})\end{array}$ & $\mathrm{MJD}_{r}$ & $\begin{array}{c}r \\
(\mathrm{mag})\end{array}$ & $\begin{array}{c}\sigma_{r} \\
(\mathrm{mag})\end{array}$ & $\mathrm{MJD}_{i}$ & $\begin{array}{c}i \\
(\mathrm{mag})\end{array}$ & $\begin{array}{c}\sigma_{i} \\
(\mathrm{mag})\end{array}$ & $\mathrm{MJD}_{z}$ & $\begin{array}{c}z \\
(\mathrm{mag})\end{array}$ & $\begin{array}{c}\sigma_{z} \\
(\mathrm{mag})\end{array}$ \\
\hline$\ldots$ & $\ldots$ & $\ldots$ & $\ldots$ & $\cdots$ & $\ldots$ & 56683.325 & 15.22 & 0.01 & $\ldots$ & $\cdots$ & $\ldots$ & $\ldots$ & $\ldots$ & $\ldots$ \\
\hline$\ldots$ & $\ldots$ & $\ldots$ & 56697.107 & 16.06 & 0.02 & 56697.108 & 15.08 & 0.01 & 56697.106 & 14.53 & 0.01 & $\ldots$ & $\ldots$ & $\ldots$ \\
\hline$\ldots$ & $\ldots$ & $\ldots$ & 56697.152 & 16.04 & 0.02 & 56697.153 & 15.07 & 0.01 & 56697.151 & 14.52 & 0.01 & $\ldots$ & $\ldots$ & $\ldots$ \\
\hline 56706.152 & 18.22 & 0.04 & 56706.15 & 16.17 & 0.01 & 56706.151 & 15.20 & 0.01 & 56706.148 & 14.66 & 0.00 & 56706.151 & 14.27 & 0.02 \\
\hline 56706.2 & 18.20 & 0.06 & 56706.197 & 16.16 & 0.02 & 56706.198 & 15.19 & 0.01 & 56706.196 & 14.64 & 0.00 & 56706.199 & 14.29 & 0.03 \\
\hline
\end{tabular}

Note. The griz photometry has been calibrated relative to SDSS stars (see the text). The $u$-band photometry has been calibrated relative to Swift UVOT observations of the same field

a $5 \sigma$ upper limit.

(This table is available in its entirety in machine-readable form.)

or 3-4 mag in $A_{V}$. Considering the large near-infrared color changes relative to the unchanging optical colors during this same time period could suggest scattering in addition to reddening. Alternately, that the near-infrared becomes redder while the optical has the same colors could be due to a dust echo.

\subsection{Mid-infrared Photometry}

The WISE satellite, after not dectecting the PTF 14jg progenitor during its main mission when observing the field in 2010 and 2011, did detect it in outburst during the warm WISE mission redubbed as NEOWISE (Mainzer et al. 2014). A strong detection was recorded on 2014 February 17 around the optical peak, with $W 1=10.83 \mathrm{mag}$ and $W 2=9.98 \mathrm{mag}$ $(W 1-W 2=0.85 \mathrm{mag}$ in color). The available NEOWISE time series (Figure 4, right panel) shows no evidence for color evolution until the last epoch, though the data are noisy. This late-time blueing seems to occur 100-200 days later than the blueing seen in the optical colors (Figure 4, left panel), which is associated with the tertiary peak in the optical light curve.

Mid-infrared photometry was also obtained with the Spitzer Space Telescope, based on a Director's Discretionary Time (DDT) allocation, over four epochs (2014 April, May, November, and December). The new data are reported in Table 5 and illustrated in Figures 3 and 4. Compared to the preoutburst Spitzer color of [3.6]-[4.5] $=0.4 \pm 0.2 \mathrm{mag}$ reported above, the outburst color was redder at $[3.6]-[4.5]=0.7 \mathrm{mag}$ but seems to have become slightly bluer as the source faded by nearly $2 \mathrm{mag}$ in the mid-infrared, reaching [3.6]-[4.5] = $0.6 \mathrm{mag}$ in the long plateau phase. If significant, this is the only color to exhibit a blueing trend in the outburst period.

Referring to Figure 3, near the optical peak, PTF 14jg was $0.1 \mathrm{mag}$ fainter in $W 1$ and $0.05 \mathrm{mag}$ brighter in $W 2$ than recorded 2 months later in S[3.6] and S[4.5], respectively. Similarly, the next NEOWISE epoch shows WI fainter and W2 brighter than the corresponding S[3.6] and S[4.5] data taken a few months later. These small magnitude differences lead to the color differences between NEOWISE and Spitzer that are apparent in Figure 4. We believe that the NEOWISE-Spitzer offsets are due to color terms that arise for red sources like PTF 14jg. Hillenbrand et al. (2018) reported a similar finding and an empirically derived relation for transforming Spitzer/ IRAC photometry to the NEOWISE filter system: $(W 1-W 2)=$ $1.62 \times(I 1-I 2)-0.04 \mathrm{mag}$, with $\mathrm{rms}=0.24 \mathrm{mag}$. This indeed brings the two colors for PTF 14jg into better agreement.

\subsection{Millimeter Photometry}

CARMA was used to observe PTF 14jg at $108 \mathrm{GHz}(2.8 \mathrm{~mm})$ on 2014 February 15 . There was no detection in the continuum at the expected source position (or anywhere within $\pm 40^{\prime \prime}$ ). The rms noise level was $0.30 \mathrm{mJy}$, and the beam size was $5.7 \times$ 3." 6. Also, ${ }^{12} \mathrm{CO}$ was not detected between velocities $v_{\mathrm{LSR}}=$ -2.6 and $-83.4 \mathrm{~km} \mathrm{~s}^{-1}$, with an rms noise level of $0.35 \mathrm{~K}$ (in $1 \mathrm{~km} \mathrm{~s}^{-1}$ channels) and a beam size of 5!" $6 \times 3$ ". 3 .

The $4 \sigma$ upper limit to the flux density of $1.2 \mathrm{mJy}$ implies an upper limit to the dust mass of $0.26 M_{\odot}$, assuming a distance of $2 \mathrm{kpc}$, "standard" disk opacities of $2.3 \mathrm{~cm}^{2} \mathrm{~g}^{-1}$ at $230 \mathrm{GHz}$ and $\beta=1$ (Beckwith et al. 1990), a gas-to-dust ratio of 100, and a dust temperature of $25 \mathrm{~K}$. If the source is located at a larger distance, then our limits are higher by the distance ratio squared.

\subsection{Low- and High-dispersion Spectroscopy}

As summarized in Table 6, a number of telescopes and instruments were used to characterize the spectroscopic evolution of PTF 14jg as it reached its photometric peak and then faded in brightness. The spectra were obtained at the Apache Point Observatory (DIS optical spectra at $R \approx 2400$, TripleSpec infrared spectrum at $R \approx 3500$ ), Palomar Observatory (DBSP optical spectra at $R \approx 2000$, TripleSpec infrared spectrum at $R \approx 3000$ ), and Keck Observatory (HIRES optical spectra at $R \approx 34,000-48,000$, DEIMOS optical spectrum at $R \approx 2200$, MOSFIRE infrared spectrum at $R \approx 3300$ ). Our first optical and infrared spectra at low dispersion were taken about a week before the peak, and the second optical spectrum was obtained about 1 week post-peak. Spectroscopic monitoring continued during the initial decline and for the next several years during the light-curve plateau phase, secondary peaks, and resumed declines.

In addition to the low-dispersion data, Table 6 also reports high-dispersion spectroscopic observations. Keck/HIRES spectra were obtained on two occasions by Howard Isaacson and processed through the standard California Planet Search image processing and spectral extraction pipeline. Four additional Keck/HIRES spectra were acquired by L.A.H. and reduced using the MAKEE package (written by Tom Barlow). The signal-to-noise ratio $(\mathrm{S} / \mathrm{N})$ values for these spectra range from 15 to 5 around $5500 \AA$ and from 35 to 15 around $7500 \AA$.

The initial PTF 14jg optical low-dispersion spectrum (see Figure 6, where it is compared to PTF 10qpf $=$ HBC 722 in outburst) showed a red continuum with prominent absorption in Ca II H \& K, the Na I D doublet, O I 7774 and $8446 \AA$, and possibly weak Ba II $6497 \AA$ A. It initially compared well to late-F 


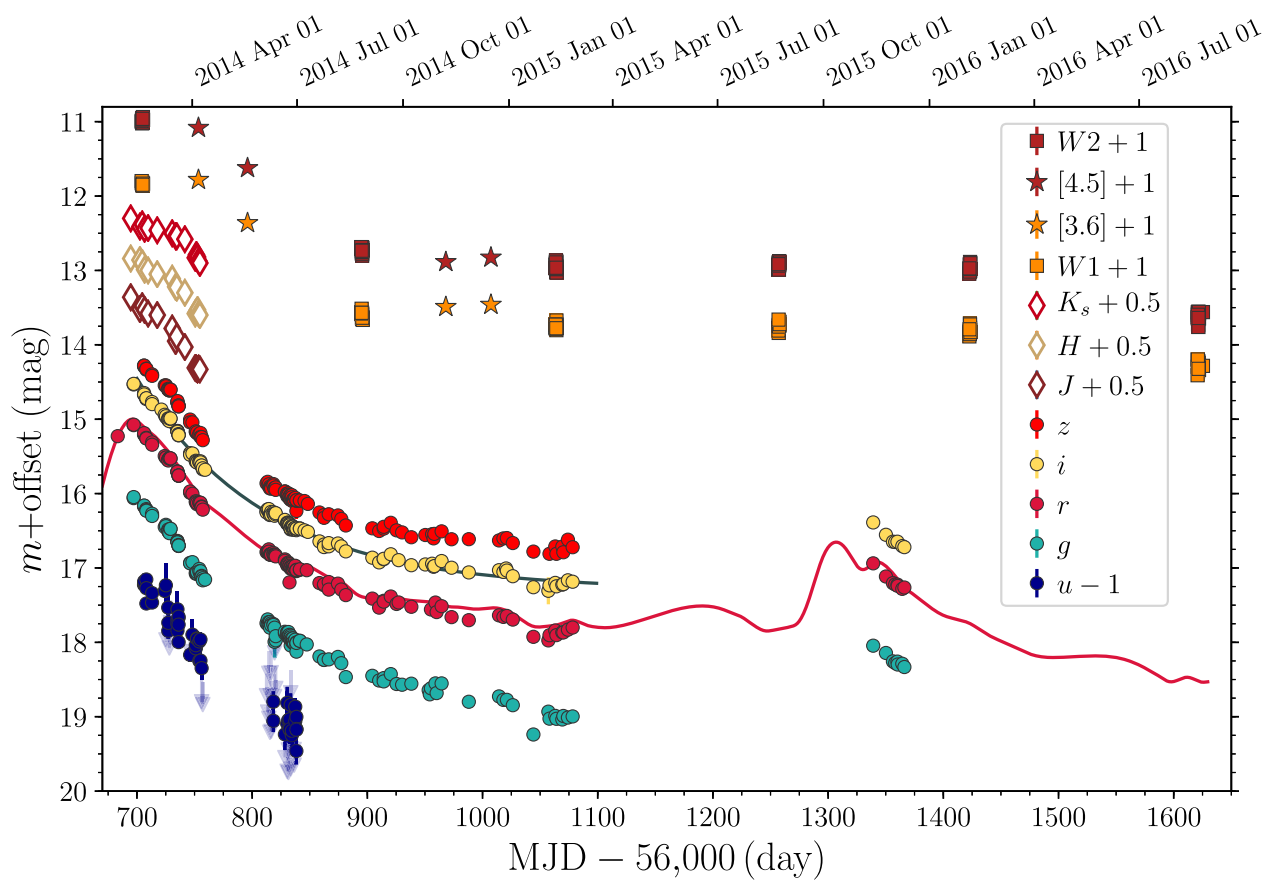

Figure 3. Post-outburst brightness evolution in the optical ugriz P60 telescope photometry, near-infrared JHK Mount Abu 1.2 m photometry, and mid-infrared IRAC1 and IRAC2 from Spitzer and W1 and W2 from NEOWISE photometry. Light curves begin in early 2014, near or just after the peak brightness of PTF 14jg. The decline over $3 \mathrm{mag}$ in brightness is relatively colorless for the duration of the time series, with color changes of no more than 0.2 mag (see details in Figure 4); the source is currently still 3-4 mag brighter than its pre-outburst state. The solid red line overlaid on the $r$-band light curve is a Gaussian-process model fit to the $R_{\mathrm{PTF}}$ photometry of Figure 1.

and G giant/supergiant templates from Silva \& Cornell (1992), with the exception of $\mathrm{H} \alpha$, which, if present, was weak and blueshifted. The Ca II "near-infrared" triplet exhibited an emission component as part of a $\mathrm{P}$ Cygni-type profile, indicating outflow. The [Ca II] doublet at 7291, $7324 \AA$ was in emission, and there was also weak emission from $\mathrm{Fe} I$ multiplet 60, strongest at $8387 \AA$, as well as Mg I $8807 \AA$.

Moderate-strength absorption from Si II 6347 and $6371 \AA$ was also apparent in this earliest outburst spectrum. Aided by the high-dispersion spectrum obtained later, we identified additional "hot" lines visible at low dispersion, specifically from, e.g., Si II 3858, 4128, 4815, 5958, 5979 Å; Mg II 4481, 5228, 5311, 5954, 5982, 6239, probable 6343 and 6366 (contributing in the Si II 6347 and 6371 regions), 6447, 7877, 7896, 8214, $8235 \AA$ A; and Fe II e.g., 4924, 5018, doublet 5169, $5316,6433 \AA$. Many of these lines have blue asymmetries in their absorption profiles.

In the earliest high-dispersion spectrum (discussed in detail below in Section 7), a confounding mix of broad and deep absorption features was present, with no readily identifiable continuum, along with the narrow emission-line component mentioned above. Examination at high dispersion of the deep lines seen in the low-resolution spectrum showed that many have strong outflow signatures. This complicated the spectrum interpretation, especially as many of the lines could not even be uniquely identified given their breadth and blueshift.

The PTF 14jg near-infrared spectra (see Figure 7) exhibited a blue continuum, in contrast to the red optical continuum. There are no detectable absorption features anywhere, but there are several narrow metallic emission lines, consistent with the presence of such lines in the optical spectrum. No forbidden line emission was seen, unlike in the optical, where [Ca II] was exhibited. Nor was there any $\mathrm{H}_{2}$ or $\mathrm{CO}$ bandhead emission (or absorption) in the infrared spectrum; these molecular features are seen in some young stars having only optical and nearinfrared continua, with few absorption lines, such as PTF 14jg.

\section{Analysis of Outburst Colors and SED}

In the optical, the (dereddened) burst colors of PTF 14jg are blue and imply ultraviolet excess relative to a normal stellar atmosphere. The colors are also much bluer than typical for young star accretion systems, and they would be on the extreme blue end of known cataclysmic variables (by $0.1-0.2 \mathrm{mag})$.

In the near-infrared, as shown in the left panel of Figure 5, the burst colors of $J-H \approx 0.45-0.75 \mathrm{mag}$ and $H-K \approx$ $0.5-0.85 \mathrm{mag}$ are red and denote an infrared excess relative to the colors expected from normal stars, in a manner that cannot be explained by reddening. PTF $14 \mathrm{jg}$ resides in the color regime populated by standard low-mass $\mathrm{T}$ Tauri stars and higher-mass Herbig Ae/Be stars with disks. The colors are similar to but slightly bluer than those of the FU Ori stars, including FU Ori itself, V1515 Cyg, and V1057 Cyg, and much bluer than those of the EX Lup stars.

The mid-infrared colors of the PTF 14jg outburst are also redder than can be explained by reddened stellar photospheres, with $K-L>2 \mathrm{mag}$, [3.6]-[4.5] $=0.7 \mathrm{mag}$, and $W 1-W 2 \approx$ $0.85 \mathrm{mag}$. In the pre-outburst phase, as mentioned above, Spitzer measured an uncertain color of [3.6]-[4.5] $\approx 0.4$, indicating moderate infrared excess (though formally only at the $2 \sigma$ level).

Figure 8 shows the available pre-outburst and outburst SED compared to model photospheres. We have already hinted that that the outburst source is spectroscopically hot, and in the next section, we present our conclusions regarding an early-type optical spectrum. The observed SED is therefore modeled in outburst with an A0 supergiant stellar atmosphere. A hot 

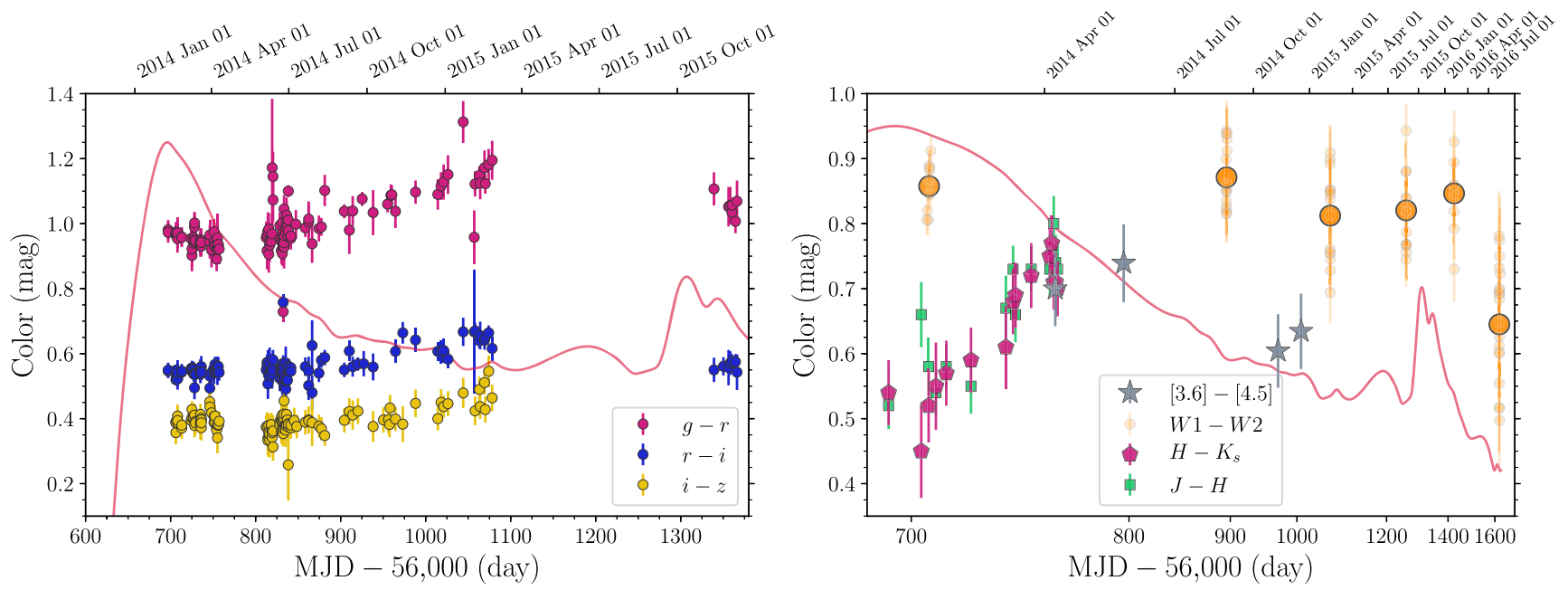

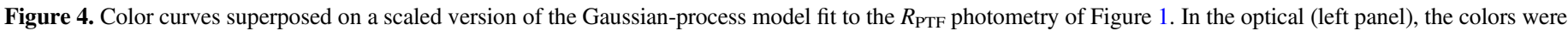

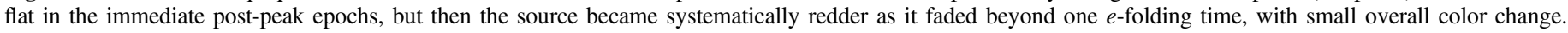

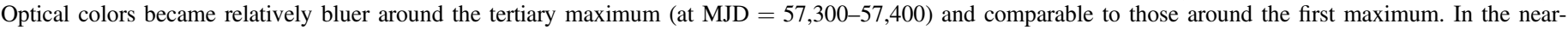

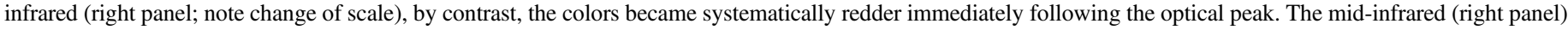

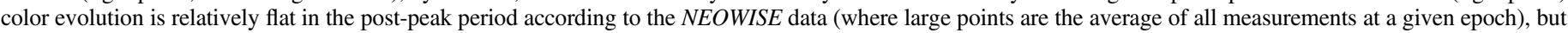
the Spitzer photometry appears to indicate blueing behavior.

Table 4

Mount Abu Near-infrared Observations

\begin{tabular}{lcccccc}
\hline \hline MJD & $\begin{array}{c}J \\
(\mathrm{mag})\end{array}$ & $\begin{array}{c}\sigma_{J} \\
(\mathrm{mag})\end{array}$ & $\begin{array}{c}H \\
(\mathrm{mag})\end{array}$ & $\begin{array}{c}\sigma_{H} \\
(\mathrm{mag})\end{array}$ & $\begin{array}{c}K_{s} \\
(\mathrm{mag})\end{array}$ & $\begin{array}{c}\sigma_{K_{s}} \\
(\mathrm{mag})\end{array}$ \\
\hline $56,694.61$ & 12.79 & 0.03 & 12.34 & 0.03 & 11.77 & 0.06 \\
$56,702.60$ & 12.95 & 0.05 & 12.36 & 0.05 & 11.88 & 0.09 \\
$56,704.59$ & 12.91 & 0.03 & 12.40 & 0.03 & 11.85 & 0.06 \\
$56,706.63$ & 12.96 & 0.03 & 12.49 & 0.03 & 11.92 & 0.09 \\
$56,709.67$ & 13.01 & 0.03 & 12.50 & 0.03 & 11.90 & 0.06 \\
$56,717.60$ & 13.03 & 0.05 & 12.55 & 0.05 & 11.93 & 0.06 \\
$56,730.60$ & 13.21 & 0.05 & 12.61 & 0.05 & 11.97 & 0.08 \\
$56,733.59$ & 13.38 & 0.03 & 12.72 & 0.03 & 12.01 & 0.06 \\
$56,734.59$ & 13.32 & 0.05 & 12.73 & 0.05 & 12.01 & 0.06 \\
$56,741.59$ & 13.46 & 0.03 & 12.80 & 0.03 & 12.05 & 0.06 \\
$56,750.61$ & 13.74 & 0.05 & 13.08 & 0.05 & 12.30 & 0.06 \\
$56,751.61$ & 13.75 & 0.05 & 13.06 & 0.05 & 12.26 & 0.06 \\
$56,752.61$ & 13.76 & 0.05 & 13.03 & 0.05 & 12.29 & 0.06 \\
$56,753.61$ & 13.75 & 0.05 & 13.08 & 0.05 & 12.34 & 0.06 \\
$56,754.61$ & 13.76 & 0.05 & 13.10 & 0.05 & 12.37 & 0.06 \\
\hline
\end{tabular}

Note. Photometry has been calibrated relative to the MKO system (see the text).

(900 K) infrared excess is also required, and in order to model the full SED, extinction of $A_{V}=4.75 \mathrm{mag}$ is implied.

We illustrate the same model fit to the pre-outburst photometry. However, the limited number of pre-outburst data points allows for a wider range of spectral type and extinction combinations to also provide a good match. Specifically, photospheres warmer than $\sim 5700 \mathrm{~K}$ with the same extinction and temperature blackbody dust excess as in outburst can also fit the photometric measurements in the $R$ band and 3.6 and $4.5 \mu \mathrm{m}$. Cooler photospheres demand less extinction, with, e.g., a $4000 \mathrm{~K}$ photosphere and no extinction plus the same temperature (but higher luminosity) blackbody excess also a good match. The nature of the pre-outburst object is not well constrained.

In the outburst phase, the shape of the 1-2.5 $\mu \mathrm{m}$ spectrum has a best-fit Planck function of temperature $T=3417 \mathrm{~K}$ for the observed spectrum and $T=9438 \mathrm{~K}$ for the spectrum dereddened using the adopted $A_{V}=4.75 \mathrm{mag}$ value. This rough color temperature estimate was already implied by the broadband SED fitting above but more directly demonstrates consistency between the hot spectral type of the source derived from spectral absorption lines in the optical (see Section 7) and the SED slope in the near-infrared.

The SED is totally unconstrained at long wavelengths. The mass upper limit from the submillimeter observations is a factor of 10 higher than the median mass of T Tauri disks. It is thus not too surprising that we did not detect the source if it contains only a disk. In the context of FU Ori stars, the upper limit is at about the median mass according to the SED modeling by Gramajo et al. (2014), which includes both envelope and disk mass.

Finally, as shown in the right panel of Figure 5, PTF 14jg is consistent with a young pre-main-sequence star at the assumed source distance, regardless of the exact reddening value.

\section{Analysis of Outburst Light Curve}

The PTF light curve covers approximately 1130 days of the PTF 14jg pre-outburst and outburst evolution. Prior to the major brightening, the source was variable about its mean magnitude at the $0.60 \mathrm{mag}$ (rms) level. After the outburst, however, variation about the fitted exponentials (see below) was only 0.09 mag (rms).

The immediate post-peak behavior of PTF 14jg (see Figure 2) was that of roughly linear decline at a rate of $\sim 0.5 \mathrm{mag}$ month $^{-1}$. After several weeks, the light curve increased its decline to $\sim 0.8 \mathrm{mag} \mathrm{month}^{-1}$, which again lasted a few weeks, as it then transitioned to a generally exponential shape. There are indications of several brief plateaus over the post-peak year. Then, between 1 and $3 \mathrm{yr}$ after the first peak, several more substantial departures from the exponential decay occurred, with evidence for two local maxima (see Figure 1). These brief rises and the exponential decays from them resulted in the PTF 14jg light curve resuming its previous exponential decline from the main peak. 

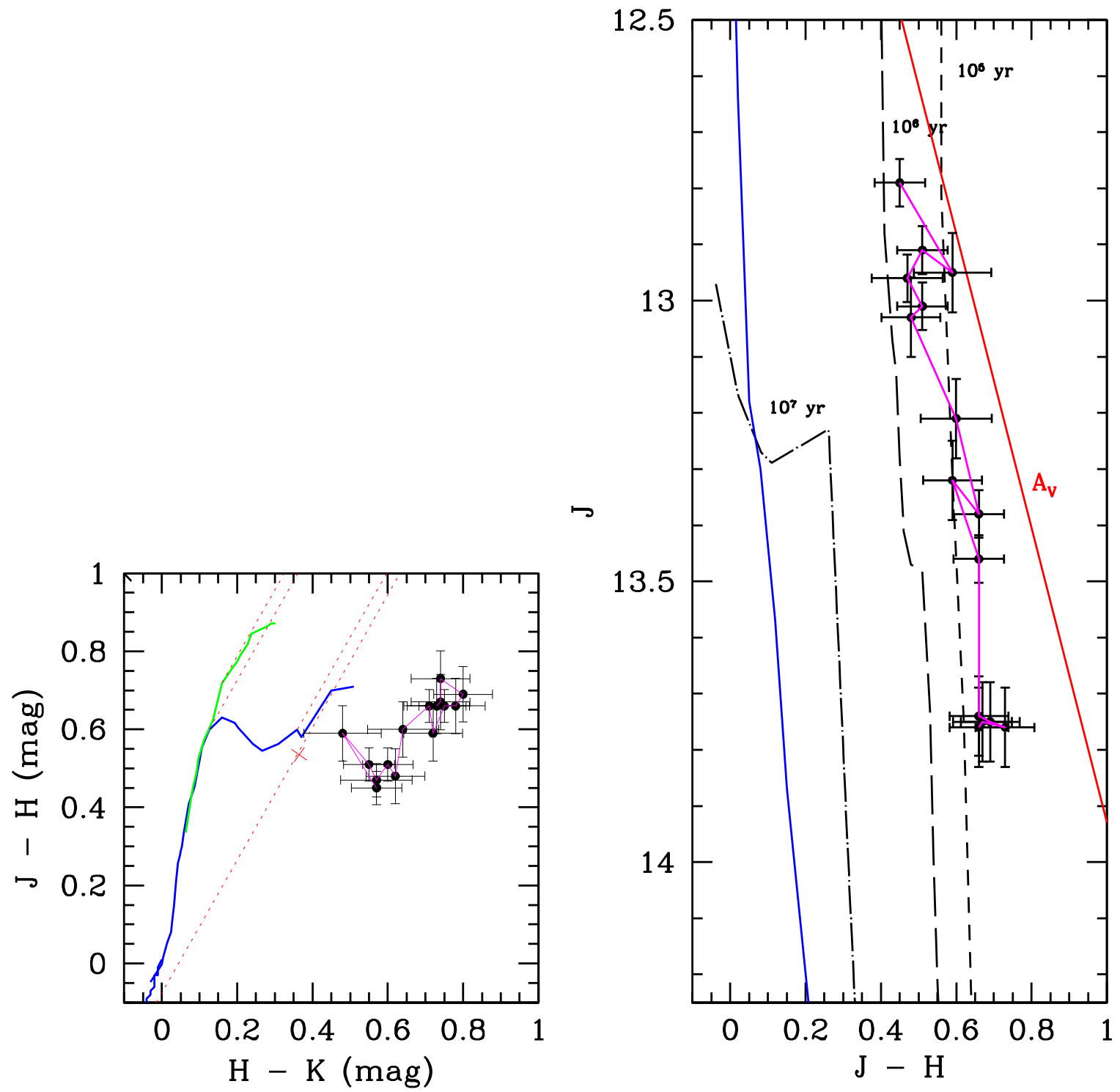

Figure 5. Near-infrared photometry in the post-peak outburst stage. The color-color diagram compares PTF 14jg to the sequence of normal dwarf and giant (blue and green solid lines) stars and illustrates the infrared excess. The color-magnitude diagram compares to pre-main-sequence isochrones assuming a $1.95 \mathrm{kpc}$ distance (black lines) and illustrates the pre-main-sequence nature of the source. Magenta lines connect the time series and show PTF 14jg generally becoming redder over time as it faded post-peak. The fade is somewhat steeper than the expectations from standard extinction (red lines), with less color change given the magnitude change.

Table 5

Spitzer Photometry

\begin{tabular}{lcccc}
\hline \hline MJD & {$[3.6]$} & $\mathrm{S} / \mathrm{N}$ & {$[4.5]$} & $\mathrm{S} / \mathrm{N}$ \\
\hline $56,753.394$ & 10.72 & 2109 & 10.03 & 2773 \\
$56,796.004$ & 11.30 & 1812 & 10.57 & 1918 \\
$56,968.136$ & 12.42 & 1015 & 11.83 & 1187 \\
$57,007.288$ & 12.39 & 1212 & 11.78 & 1213 \\
\hline
\end{tabular}

Using traditional nova nomenclature, the post-peak times $t_{1}$, $t_{2}$, and $t_{3}$ (corresponding to the times at which the light curve has declined from its maximum by 1,2 , and $3 \mathrm{mag}$, respectively) are 54, 139, and 747 days. These are much longer timescales than typical of the standard nova categories. Regarding the light-curve shape, PTF $14 \mathrm{jg}$ would be similar to a P-class or plateau nova, but it also exhibits several C-classtype rebrightenings, albeit with very different shapes compared to typical cusp novae (see Strope et al. 2010).
We fit the pre-outburst and outburst rise with a sigmoid function, coming to a peak of $R_{\mathrm{PTF}}=14.96 \mathrm{mag}$ at a time $t=0$, and the post-peak decay with an exponential. The sigmoid has parameters $L /\left(1+e^{\left(-k \times\left(t-t_{o}\right) / \text { days }\right)}\right)$. For the first peak, our best-fit values are $t_{o}=-56.23 \pm 0.52$ (meaning that the outburst starts $2 \times t_{o}=112.5$ days before its peak), $k=$ $-0.066 \pm 0.002$ (where the $1 / k$ value represents a timescale for the rise, a 15.2 day $e$-folding), and $L=6.73 \pm 0.04$ (representing the amplitude of the rise in magnitude). The exponential decay has parameters $a \times e^{-b \times t / \text { days }}+c$, with our best-fit values for the first peak $a=-2.92 \pm 0.01, b=$ $0.0090 \pm 0.0001$, and $c=2.72 \pm 0.01$. The $a$ value indicates the post-peak decline in magnitudes. The $1 / b$ value represents a timescale of 111 days for an $e$-folding.

The additional brightening and exponential decay that is well sampled in the PTF data, called the tertiary peak above, was also fit. The resulting values are similar to those above, with $a=$ $-2.34 \pm 0.03, b=0.0058 \pm 0.0002$, and $c=2.15 \pm 0.04$. The 
Table 6

Summary of Spectroscopic Observations

\begin{tabular}{|c|c|c|c|c|c|}
\hline $\begin{array}{l}\text { UT Date } \\
\text { (YYYY MM DD) }\end{array}$ & $\begin{array}{l}\text { MJD } \\
\text { (day) }\end{array}$ & $\begin{array}{l}\text { Post-peak } \\
\quad \text { (day) }\end{array}$ & $\begin{array}{l}R_{\mathrm{PTF}} \\
(\mathrm{mag})\end{array}$ & Telescope/Instrument & Observers/Reducers \\
\hline \multicolumn{6}{|l|}{ Optical Spectra } \\
\hline 20140203 & 56691 & -6 & 15.05 & APO/DIS & M. Kasliwal/Y. Cao \\
\hline 20140218 & 56706 & 9 & 15.11 & APO/DIS & M. Kasliwal/Y. Cao \\
\hline 20140221 & 56709 & 12 & 15.15 & Keck/HIRES & H. Isaacson et al. \\
\hline 20140225 & 56713 & 16 & 15.20 & Palomar $200 \mathrm{inch} / \mathrm{DBSP}$ & A. Waszczak \\
\hline 20140301 & 56717 & 20 & 15.25 & Keck/DEIMOS & S. Tang \& Y. Cao \\
\hline 20140324 & 56740 & 43 & 15.73 & Palomar $200 \mathrm{inch} / \mathrm{DBSP}$ & A. Waszczak \\
\hline 20140404 & 56751 & 54 & 15.99 & Palomar $200 \mathrm{inch} / \mathrm{DBSP}$ & A. Waszczak \& A. Miller \\
\hline 20140624 & 56832 & 135 & 16.92 & Palomar $200 \mathrm{inch} / \mathrm{DBSP}$ & I. Arcavi \\
\hline 20140822 & 56891 & 194 & 17.36 & Keck/HIRES & H. Isaacson et al. \\
\hline 20141123 & 56984 & 287 & 17.54 & Palomar 200 inch/DBSP & L. Hillenbrand \& A.M. Cody \\
\hline 20141126 & 56987 & 290 & 17.54 & APO/DIS & M. Kasliwal/Y. Cao \\
\hline 20141209 & 57000 & 303 & 17.55 & Keck/HIRES & L. Hillenbrand \\
\hline 20150723 & 57226 & 529 & 17.67 & Palomar 200 inch/DBSP & Pavanman/Khazov \\
\hline 20150724 & 57227 & 530 & 17.68 & Keck/HIRES & L. Hillenbrand \\
\hline 20151027 & 57322 & 625 & 16.91 & Keck/HIRES & L. Hillenbrand \\
\hline 20151206 & 57362 & 665 & 17.18 & Palomar $200 \mathrm{inch} / \mathrm{DBSP}$ & Lunnan, Bladgorodnova/Cao \\
\hline 20160203 & 57421 & 724 & 17.86 & Keck/HIRES & L. Hillenbrand \\
\hline 20160826 & 57626 & 929 & 18.48 & Palomar 200 inch/DBSP & Cook/Knezevic \\
\hline 20171111 & 58068 & 1371 & $\cdots$ & Palomar 200 inch/DBSP & Ho, Kulkarni \\
\hline \multicolumn{6}{|l|}{ Infrared Spectra } \\
\hline 20140202 & 56690 & -7 & 15.08 & APO/TSpec & M. Kasliwal/S. Tang \\
\hline 20140323 & 56739 & 43 & 15.71 & Palomar/TSpec & Y. Cao, D. O'Sullivan/J. Jencson \\
\hline
\end{tabular}

amplitude is smaller than the main peak by $0.5 \mathrm{mag}$, and the $1 / \mathrm{b}$ timescale is longer at 173 days.

\section{Analysis of Outburst Spectroscopy}

The low-resolution optical and infrared spectra were shown in Figures 6 and 7. Portions of the first optical high-dispersion spectrum appear in Figures 9 and 10, the latter highlighting the velocity profiles of particularly illustrative lines. Subsequent figures show the spectral evolution as PTF 14jg initially cooled from its light-curve peak.

\subsection{Radial Velocity}

We took advantage of the presence of narrow emission lines in the first high-dispersion spectrum to estimate a radial velocity for PTF 14jg, which was not discernable otherwise from the broad absorption lines. To find an approximate velocity, we inverted the PTF 14jg spectrum and compared it to the $\mathrm{G}$ supergiant HR 8414 with $\mathrm{HRV}=6.63 \mathrm{~km} \mathrm{~s}^{-1}$ (Soubiran et al. 2008). The measured heliocentric velocity was $-26.6 \mathrm{~km} \mathrm{~s}^{-1}$, assuming that the weak narrow-line emission spectrum is located at the systemic velocity of the star. This value guided our spectral analysis efforts for some time.

However, a reasssessment of the radial velocity became possible when a less wind-dominated absorption-line spectrum emerged by the time of the 2015 October 27 high-dispersion spectrum. At that point, with a narrower absorption-line spectrum presented, we were able to compare to an accepted set of true radial velocity standards that we have used in our studies of eclipsing binaries. We derived $-38.1 \pm 1.2 \mathrm{~km} \mathrm{~s}^{-1}$ using standards in the FGK spectral type range. This more robust radial velocity value is entirely consistent with the location of PTF 14jg in the Perseus spiral arm.

\subsection{Spectral Type: A Composite Spectrum}

As described above, the early low-resolution optical spectrum (Figure 6) appeared roughly consistent with a reddened late-F or early- $\mathrm{G}$ supergiant spectrum. This assessment was based on the classical signature of roughly equal strength $\mathrm{Ca}$ II $\mathrm{H}$ and $\mathrm{K}$ lines, which are also stronger than the very weak Balmer HI lines, and the presence of weaker metallic features plus $\mathrm{CH}$ near $\mathrm{H} \gamma$. The lack of strong hydrogen appeared to exclude types earlier than late F. All K and later low-gravity spectral types were discounted based on the lack of expected strong absorption features from, e.g., Ca I for the K or $\mathrm{TiO}$ for the $\mathrm{M}$ star ranges. A luminosity classification of I was justified by details such as the lack of the $4700 \AA$ absorption expected from luminosity class III FG stars and the presence of features such as 6238 and $6486 \AA$ that are seen in luminosity class I but not III FG stars. However, early A- and B-type features were also present in the spectrum, including the numerous Fe II, Mg II, and Si II features listed earlier. Given the evidence for a hot-temperature spectral component, it was somewhat surprising that there is no Balmer jump visible in the low-dispersion data, and that the $\mathrm{H}$ I lines are generally absent or very weak. Overall, the spectrum of PTF 14jg eluded strict classification.

Further consideration of the PTF 14jg spectral type based on high-dispersion spectroscopy (Figure 9) only exacerbated our confusion about the nature of the source. The first Keck/ HIRES spectra showed some features that are seen only at very low gravity in early-type supergiant and giant standards, specifically those characteristic of late-F supergiants, but also feature more similar to those of late-B supergiants (B6-A0). However, these lines are much stronger in PTF 14jg than in standard stars, which we now attribute to enhanced line contributions from the outflow (see, e.g., right panels of 


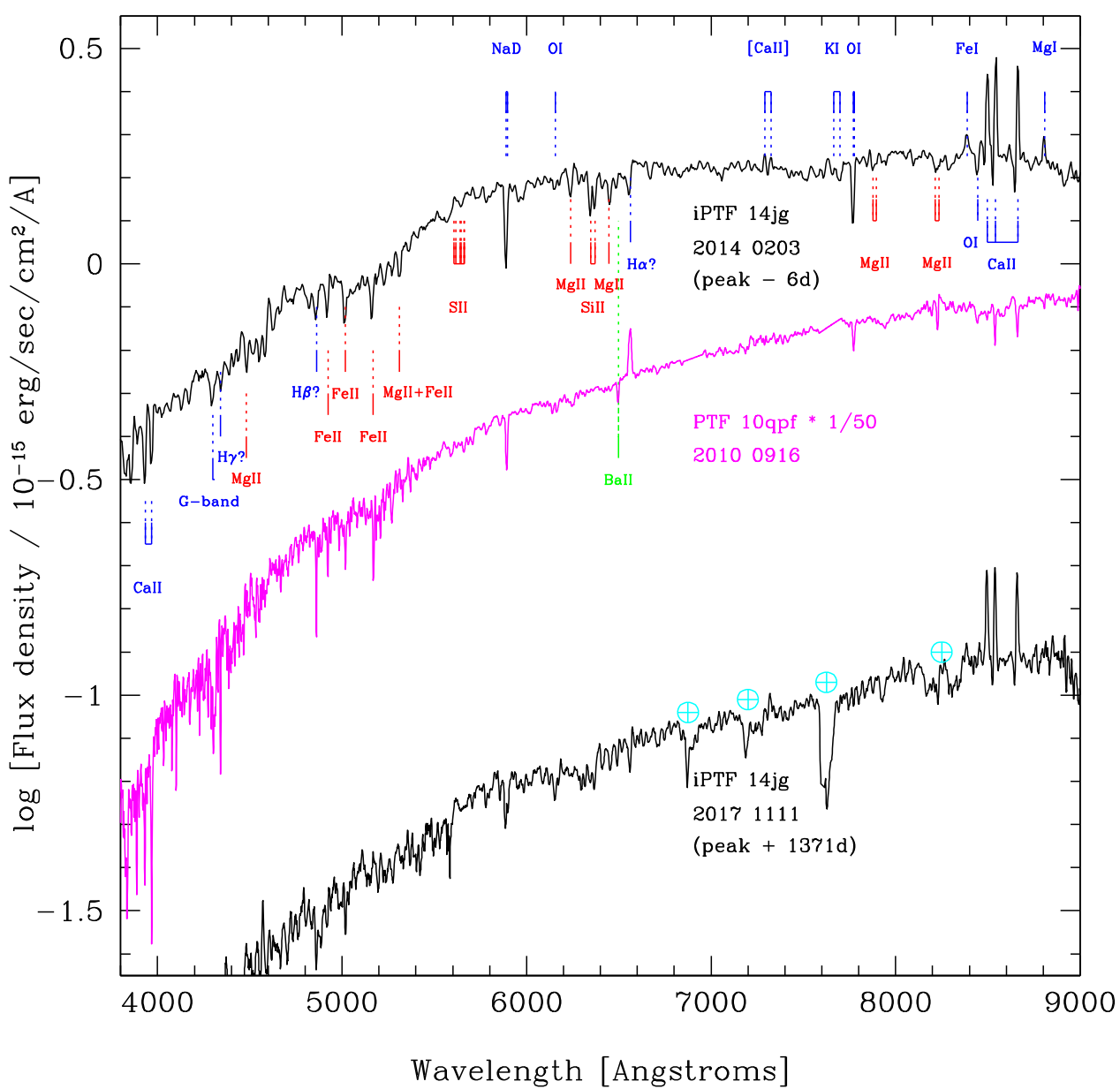

Figure 6. Comparison of the PTF 14jg low-dispersion optical spectrum with that of PTF 10qpf (HBC 722), a bona fide FU Ori star. Denoted spectral features are in blue when seen in young stars of various categories and in red if not typically seen in young stars. The low-ionization Ba II/Ca I/Fe I blend of lines at $6497 \AA$ that is commonly seen in FU Ori stars but weak in the hotter spectrum of PTF 14jg (and may in fact be an Fe II line) is also indicated. This first PTF 14jg spectrum, exhibiting red continuum, weak hydrogen lines, and ionized metal absorption components, initially appeared to be a reasonable, albeit imperfect, match to stars in the temperature range of $\mathrm{F}$ and $\mathrm{G}$ stars. However, there was additional absorption, notably within $6200-6500 \AA$, that is not explained by such cool photospheres; the 4900-5200 ^ absorptions are likewise better attributed to hotter ionized lines than the cooler neutral lines of FG stars. The bottom spectrum, taken nearly 4 yr after the initial rise, illustrates the nearly colorless optical fade yet still shows hot ionized line absorption. Telluric absorption regions are marked in cyan. The PTF14jg spectra are available as the data behind the figure. The data used to create this figure are available.

Figure 10). In the early-epoch data, particularly toward the blue, the broad and deep lines were not resolved from one another, even at high dispersion, and therefore were not readily identifiable. Many of these "hot" lines exhibiting prominent outflow signatures persisted as narrower absorption features later, as the source faded photometrically and the outflow velocities decreased.

In an attempt to decipher the spectrum, we cross-correlated with a suite of spectral template stars taken with the same Keck/HIRES settings as our PTF 14jg spectra. There was only weak correlation with KM-type supergiants or dwarfs but decent correlation power with FG supergiant standards. We also correlated PTF 14jg with a grid of BA supergiants, giants, and dwarfs taken from the Elodie grid, since we do not possess our own standards for these earlier types. For supergiants and giants, the peaks were highest overall for types in the early-A to late-F spectral range, with a clear trend with wavelength. Blue wavelengths correlated best with early-A stars, green wavelengths correlated best with late-F stars, and red wavelengths best matched GK-type stars. Figure 11 shows the correlation strengths at a much later epoch, when the strong outflow signatures had somewhat subsided, and the spectrum may have cooled as the light curve declined; however, the basic patterns described above for the early-epoch spectrum are even more apparent.

No line absorption at all was seen in the early infrared spectra (Figure 7). Thus, we cannot derive an infrared spectral type near the burst peak. Despite the blue continuum, there are no hydrogen Paschen or Brackett series lines, consistent with the lack of strong Balmer lines in the optical spectrum. Likewise, there is an absence of strong spectral features from cool-temperature gas, such as CO absorption.

There is no overall best-fitting spectral type for the early outburst spectrum of PTF 14jg. Instead, the optical spectrum exhibits evidence of a composite absorption spectrum, with a temperature that changes with wavelength. The other dominant aspect is a multitemperature wind, with both cool species, e.g., $\mathrm{Ca} \mathrm{H} \& \mathrm{~K}$ and $\mathrm{Na} \mathrm{D}$, as well as hot species, such as Fe II, Mg II, and Si II seen in blueshifted absorption. There is very little H I or He I signature apparent in PTF 14jg; the only strong absorption is from wind-produced metal lines. 


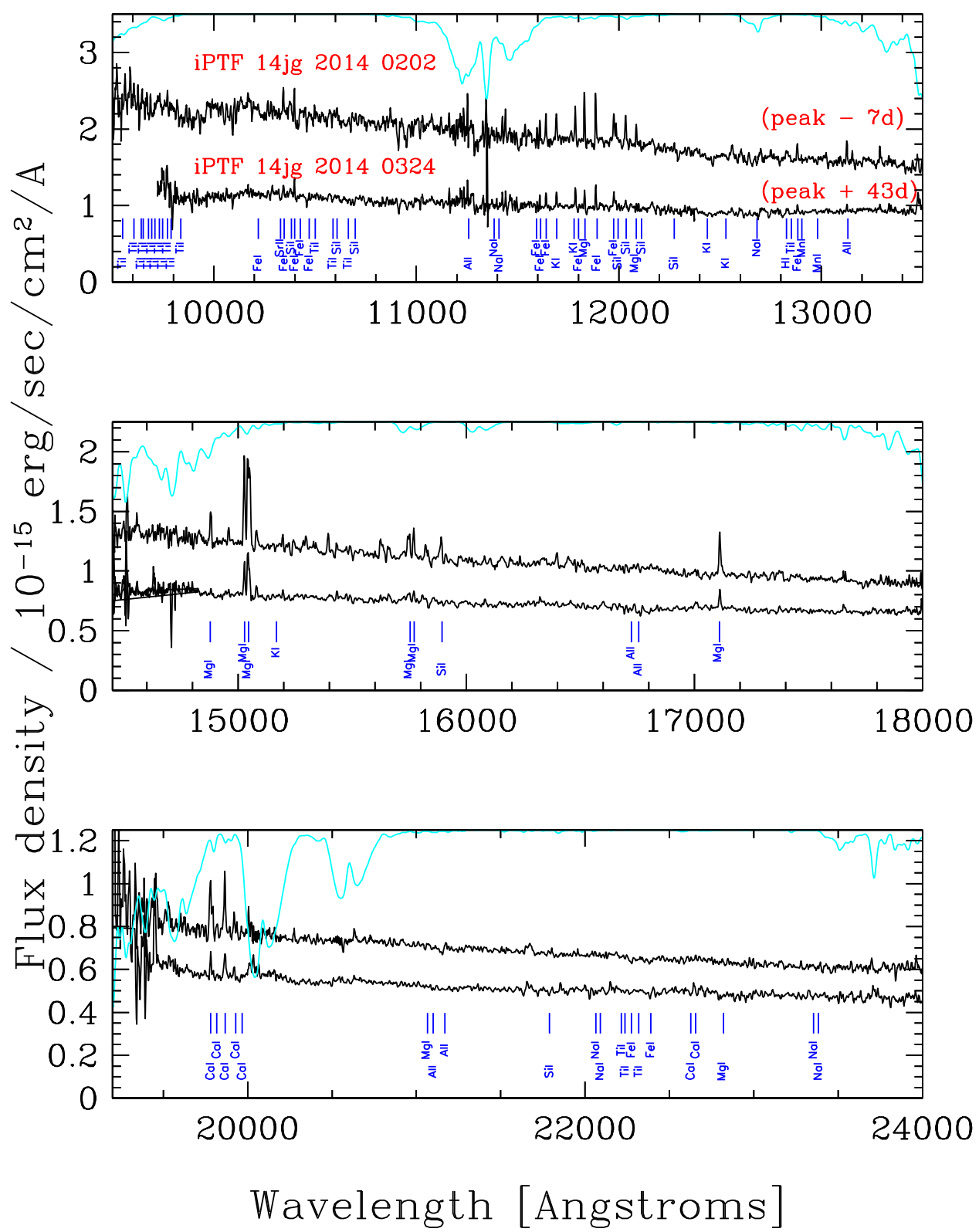

Figure 7. PTF 14jg infrared spectrum at two early epochs, illustrating a blue continuum with superposed narrow emission lines (primarily Mg I, Ca I, and Fe I) that faded within less than 6 weeks. For reference, the atomic spectral lines that are seen in the cool stars presented by Cushing et al. (2005) are marked in blue; there are no identifiable molecular features. The cyan line is a model atmospheric transmission spectrum plotted on a vertical scale from $0 \%$ to $100 \%$ and indicates regions where the telluric correction applied to the data is large and possibly uncertain. The spectra are available as the data behind the figure. The data used to create this figure are available.

\subsection{Broad Hot Absorption Spectrum}

As noted above in our attempts at spectral typing, many of the spectral features in PTF 14jg seem atypical for a singletemperature normal stellar photosphere. The initial high-dispersion optical spectrum (Figure 9) exhibited very strong lines, having equivalent widths in most lines exceeding $1 \mathrm{~A}$, and highly broadened lines, having typical FWHM $\sim 100-150 \mathrm{~km} \mathrm{~s}^{-1}$.

Some features associated with late-F supergiants could be identified, such as higher-excitation (3-4 eV) lines of Fe I and $\mathrm{Ni} I$ and the $11 \mathrm{eV}$ lines of Fe II. However, there is a preponderance of even hotter lines, such as are seen in late-B stars. The O I $8446 \AA$ singlet at $9.5 \mathrm{eV}$ is present. And we were able to associate some lines with $\mathrm{Si}$ I (intermediate excitation potential of $6 \mathrm{eV}$, e.g., 7405, 7409, 7415, and $7742 \AA$ lines);
Si II, Mg II, or Fe II (high excitation of 10.5-12 eV); and even Si III (19-20 and $26 \mathrm{eV}$, e.g., 4552, 4567, 4574, and $4716 \AA$ lines)! Placing an upper bound on the temperature, there is no Si IV at, e.g., 4088, 4116, and $4212 \AA$ ( 24 and $35-40 \mathrm{eV}$ lines).

The hot spectrum lines seem to indicate an 11,000 $15,000 \mathrm{~K}, \log g=2-3$ photospheric component of the spectrum, as assessed using the MILES stellar population synthesis tool (Vazdekis et al. 2003). From Si III/Si II ratios and guided by Lefever et al. (2010), a temperature of $15,500 \mathrm{~K}$ is inferred for the absorption spectrum. These temperature values are consistent with the cross-correlation analysis in the bluer parts of the spectrum, discussed above.

We conclude that the blue-wavelength absorption spectrum of PTF $14 \mathrm{jg}$ has a moderately warm temperature and low 

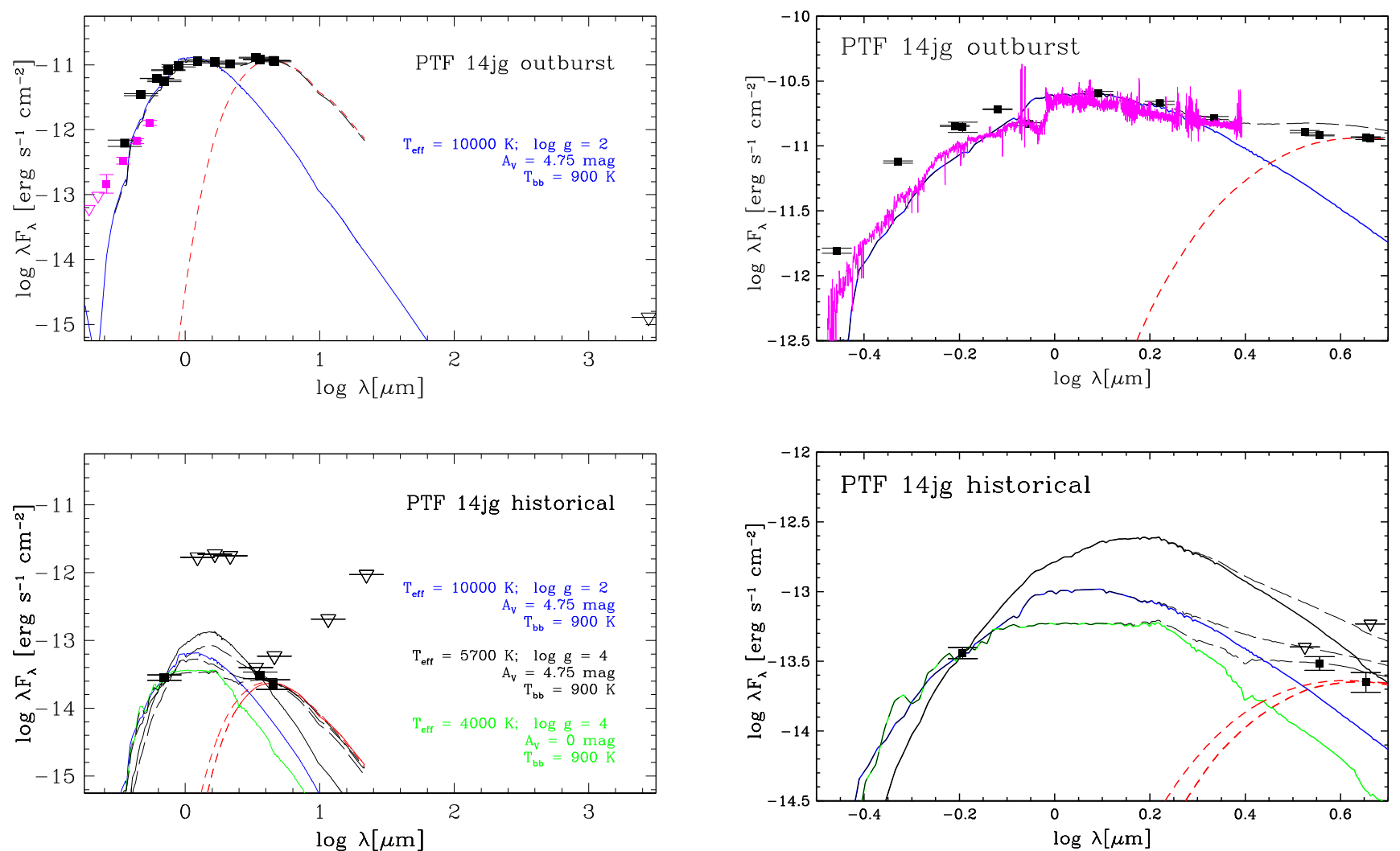

Figure 8. Post-outburst (top panels) and pre-outburst (bottom panels) SED compared to reddened model photospheres allowing for a blackbody infrared excess. The left panels have the same axis scaling. The right panels are an expanded view around the SED peak, with the magenta line showing the outburst optical and nearinfrared spectrophotometry. Filled symbols are measurements (from UVOT, P60, P48/PTF, Mount Abu, and NEOWISE-R in outburst and P48/PTF and Spitzer in the pre-outburst phase), while open triangles are upper limits (from CARMA in outburst and WISE and 2MASS in the pre-outburst phase). Blue lines represent the hot supergiant extincted photosphere that is indicated by the outburst spectrum. In the bottom panels, the black line is the minimum temperature photosphere that can still match the pre-outburst SED at the same value of extinction. The green line in the bottom panels represents a much cooler photosphere that can fit the data only if the extinction is also much lower, with the minimum case of 0 mag shown. Red lines indicate a $900 \mathrm{~K}$ blackbody matched to the longest-wavelength data point.

surface gravity. However, it is possible that much of the temperature and surface gravity information we have inferred from the absorption spectrum actually characterizes the windlaunching region, rather than a stellar or disk photosphere.

Supporting this is that the observed hot absorption lines are very deep, reaching between $90 \%$ and $70 \%$ of the continuum instead of the typical $99 \%-95 \%$ of the continuum for normal yellow supergiants/hypergiants (specifically those in the Elodie archive). In addition, many of the hot lines are affected by outflow kinematics and even broader than the $100-150 \mathrm{~km} \mathrm{~s}^{-1}$ FWHM that we believe characterizes lines defining the at-rest photosphere. For example, the Si II 6347 and $6371 \AA$ equivalent widths are off the scale of the temperature calibration by Miroshnichenko et al. due to the influence of the strong outflow (even accounting for probable contamination on the blue side by the nearby Mg II $6343+$ $6366 \AA$ lines).

\subsection{Blueshifted Absorption Features Indicative of a Wind}

Strong signatures of outflowing material are seen in many spectral lines. The descriptions below are based on the first high-dispersion spectrum, taken 12 days past maximum light, corrected for the inferred $-38.1 \mathrm{~km} \mathrm{~s}^{-1}$ systemic velocity. Figures 9 and 10 are relevant to the discussion.

The Ca II $\mathrm{H} \& \mathrm{~K}$ doublet lines are saturated and highly blueshifted. The saturated component extends from about
-125 out to $-400 \mathrm{~km} \mathrm{~s}^{-1}$, departing the continuum at all velocities between about -100 and $-450 \mathrm{~km} \mathrm{~s}^{-1}$. A separate narrow component located at zero velocity also reaches zero intensity. The $8498,8542,8662 \AA$ triplet lines, which share upper levels with the doublet lines, are in emission. Although not covered in the first two HIRES observations, in later data, these lines peak blueward of the rest velocity, around $-40 \mathrm{~km} \mathrm{~s}^{-1}$, and extend to $-200 \mathrm{~km} \mathrm{~s}^{-1}$. While the earlyepoch low-dispersion data exhibit P Cygni line structure in the triplet lines, none of our (later) high-dispersion data capture this structure.

The Na I D doublet lines are saturated between -125 and $-200 \mathrm{~km} \mathrm{~s}^{-1}$ and also have multiple unsaturated absorption components extending out to about $-400 \mathrm{~km} \mathrm{~s}^{-1}$. Like in the $\mathrm{Ca}$ II $\mathrm{H} \& \mathrm{~K}$ lines, there is a separate narrow component at zero velocity that also reaches zero intensity.

The K I $7699 \AA$ line (the companion $7665 \AA$ line is between spectral orders) exhibits two weak and broad blueshifted absorption components that seem to correspond to components at similar velocities in $\mathrm{NaD}$, extending to $-450 \mathrm{~km} \mathrm{~s}^{-1}$. There is also a strong narrow zero-velocity component, but unlike in $\mathrm{Ca}$ II $\mathrm{H} \& \mathrm{~K}$ and $\mathrm{Na}$ I $\mathrm{D}$, it is unsaturated.

The Li I $6707 \AA$ line has a broad absorption that appears asymmetric toward the blue, but the interpretation is complicated by the narrow and weak $\mathrm{Fe}$ I emission that is nearby. Several other lines appear to have a similar shallow and highly blueshifted component to their profiles between -300 and 

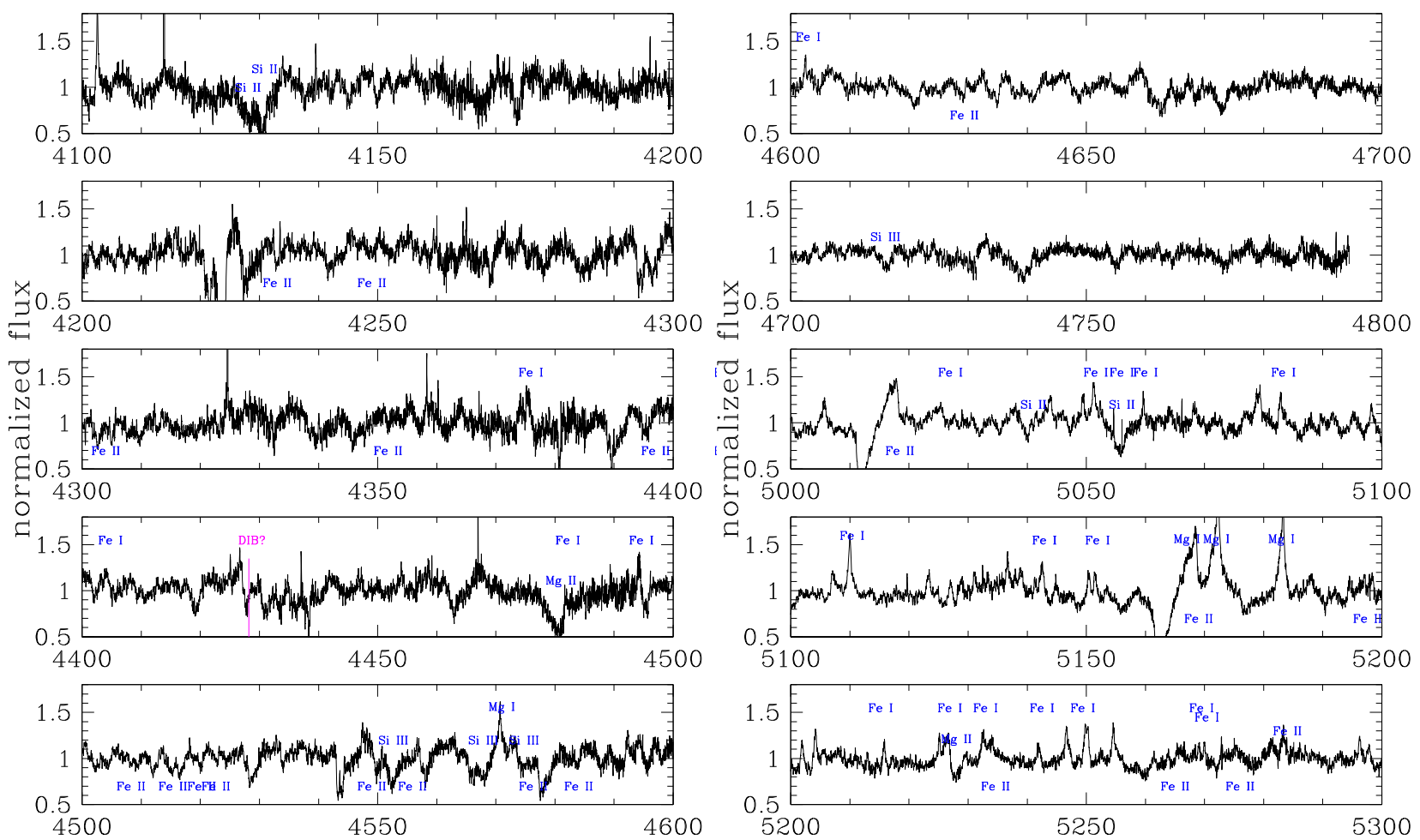

wavelength [Angstrom]
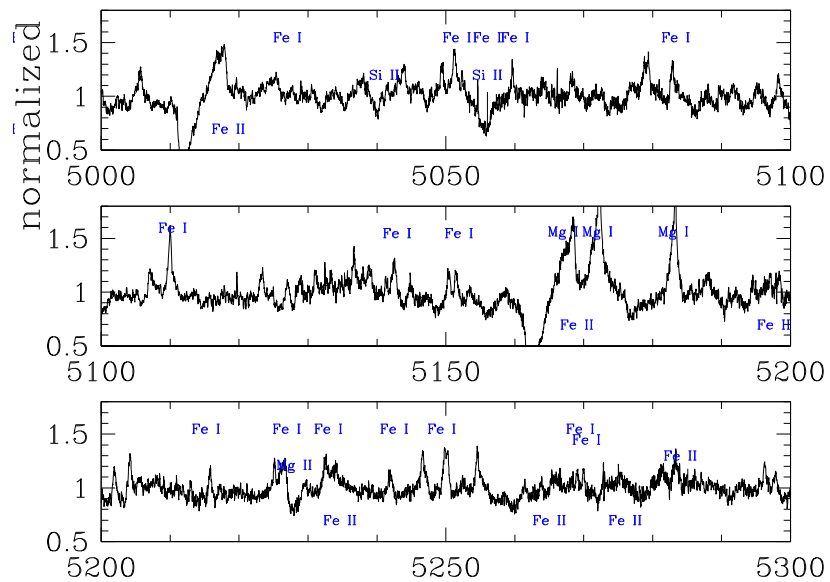

wavelength [Angstrom]
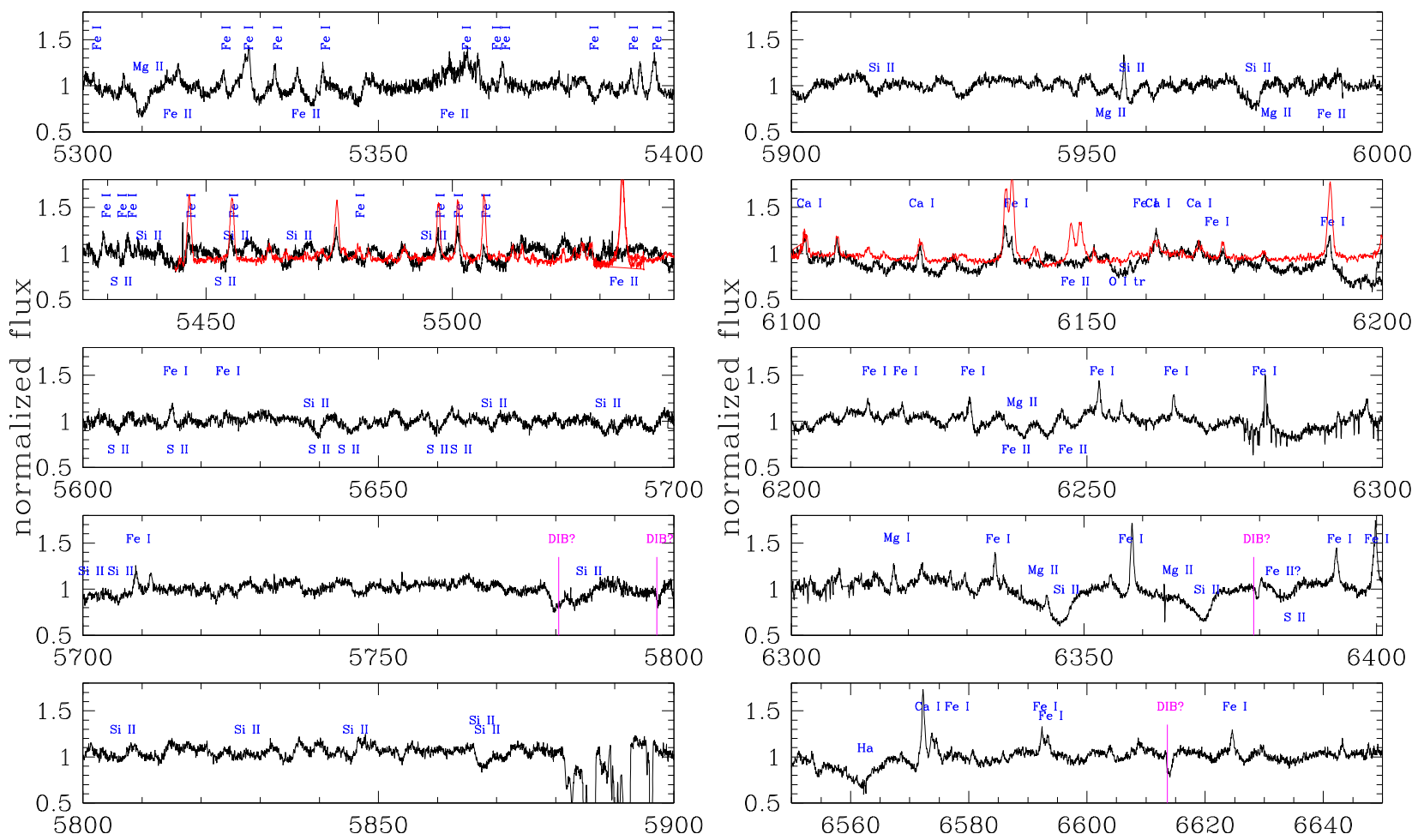

wavelength [Angstrom]

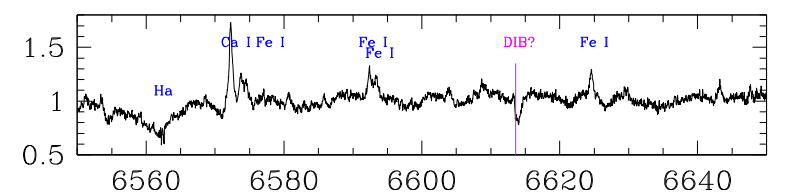

wavelength [Angstrom]

Figure 9. Portions of the first high-dispersion spectrum of PTF 14jg taken on 2014 February 21, or 12 days after the estimated light-curve peak. We note the broad and deep absorption, especially toward the blue end of the spectrum, and the superposed narrow emission lines. The well-known continuum+emission-line young stellar object V1331 Cyg is also shown in several orders (in red) for comparison. PTF 14jg shares the neutral species emission but not the (higher-excitation) ionized emission that is exhibited by V1331 Cyg. Many of the absorption lines in PTF 14jg are blueshifted from the indicated line center position. Some DIBs are present. We have been unable to identify all of the broad absorption and narrow emission contributors to this spectrum. 


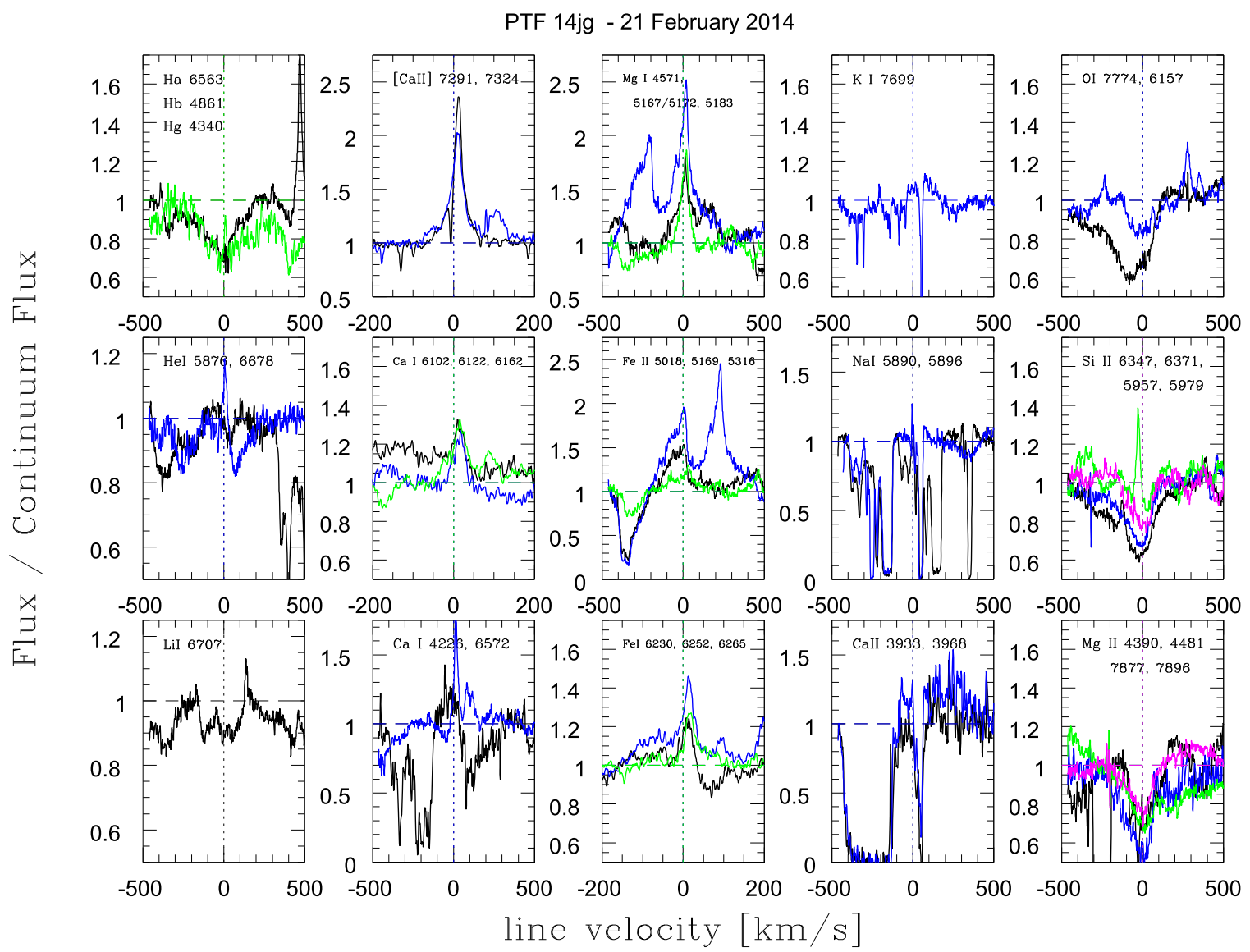

Figure 10. Line profiles from the first epoch of high-dispersion data taken 12 days after the light-curve peak. Where multiple profiles are overlaid, the color corresponds to the denoted line in order of black, blue, green, and magenta. The normalization is based on the values at the edges of the velocity range and is nonoptimal in some cases. A heliocentric velocity shift of $38.1 \mathrm{~km} \mathrm{~s}^{-1}$ has been applied so as to bring the lines to zero velocity relative to the absorption-line spectrum that developed later. Note that the narrow-peak emission-line spectrum (represented here in, e.g., Ca I, Fe I, and Mg I, as well as [Ca II]) would require a shift of only $26.6 \mathrm{~km} \mathrm{~s}^{-1}$; i.e., the narrow emission lines have a redshift of about $11.5 \mathrm{~km} \mathrm{~s}^{-1}$ with respect to the systemic velocity of the photosphere.

$-450 \mathrm{~km} \mathrm{~s}^{-1}$ (in addition to the K I already discussed; see also the description below of the $\mathrm{H} \alpha$, He I 5876, O I 7774, and Fe II 5018, 5169 lines). We are thus inclined to believe that we are seeing outflow signatures in $\mathrm{Li}$ I as well. The velocity-centered part of the line is very broad, FWHM $=200 \mathrm{~km} \mathrm{~s}^{-1}$. By 2014 December, a narrower component to the LiI profile had developed, consistent with the developing narrow $\mathrm{H} \alpha$. In 2016 February, a double-peaked Li I absorption line was seen.

The Ca I $4226 \AA$ line has multiple absorption components from -150 all the way out to $-400 \mathrm{~km} \mathrm{~s}^{-1}$. The profile appears quite similar to the Ca II H \& $\mathrm{K}$ and Na I D profiles but is not saturated. It is also similar to the multiple K I 7699 and possibly Li I 6707 profile components. The other ground-state transition of $\mathrm{Ca}$ I is at $6572 \AA$; this profile lacks the blueshifted absorption of the $4226 \AA$ line, exhibiting only narrowly peaked emission that is also seen in the weaker precursor 6102,6122 , and $6162 \AA$ lines.

The MgI 5172, 5183, and MgI $4571 \AA$ lines all show blueshifted emission between about zero velocity and $-200 \mathrm{~km} \mathrm{~s}^{-1}$. The $5167 \AA$ component of the Mg I 5167, 5172,5183 triplet is confused with the $5169 \AA \mathrm{Fe}$ II line discussed below. The $4571 \AA$ line is a singlet that immediately follows the triplet in cascade to the ground state. These lines, as well as the $8806 \AA$ line that is seen in the low-dispersion spectrum (but not covered by HIRES), are of intermediate excitation, in the $3-6 \mathrm{eV}$ range.
The O I $7774 \AA$ triplet is very broad, extending beyond $-500 \mathrm{~km} \mathrm{~s}^{-1}$ on the blue end and redward to about $+75 \mathrm{~km} \mathrm{~s}^{-1}$. However, as the velocity scale is set at the central wavelength of the middle triplet component, the extreme red and blue velocities compared to other features are due to the presence of the multiplet. The O I $6157 \AA$ triplet line seems narrower; its three lines span only $2.2 \AA$ rather than $3.5 \AA$ for the $7774 \AA$ triplet.

The Fe II 5018 and $5169 \AA$ lines are similar to the $\mathrm{Mg}$ I in showing blueshifted emission between about zero velocity and $-200 \mathrm{~km} \mathrm{~s}^{-1}$. In addition, there is deep absorption between -200 and $-450 \mathrm{~km} \mathrm{~s}^{-1}$, reaching maximum absorption strength at -350 to $-400 \mathrm{~km} \mathrm{~s}^{-1}$. These are low-excitation $(3 \mathrm{eV})$ iron lines. A higher-excitation $(10 \mathrm{eV})$ line at $5316 \AA$ has similar high-velocity structure but is much weaker.

The Si II 6347, 6371 ̊, 3853, 3856, $3862 \AA, 4128,4130 \AA$, and $4815,5041,5056,5957,5978 \AA$ lines are predominantly blueshifted with a wing out to $-450 \mathrm{~km} \mathrm{~s}^{-1}$ but have absorption extending redward of line center. These are all high-excitation $(8-10 \mathrm{eV})$ lines that are not commonly seen in young star winds. They are present in supernova spectra, however, and suggest that a hot shell-like feature may be associated with the photometric brightening of PTF 14jg. No lines such as $\mathrm{C}$ I with similar excitation potential are prominent in our spectra.

The Mg II 4481， 7877, $7896 \AA$ lines are similar in morphology to the Si II lines, which is not surprising given 


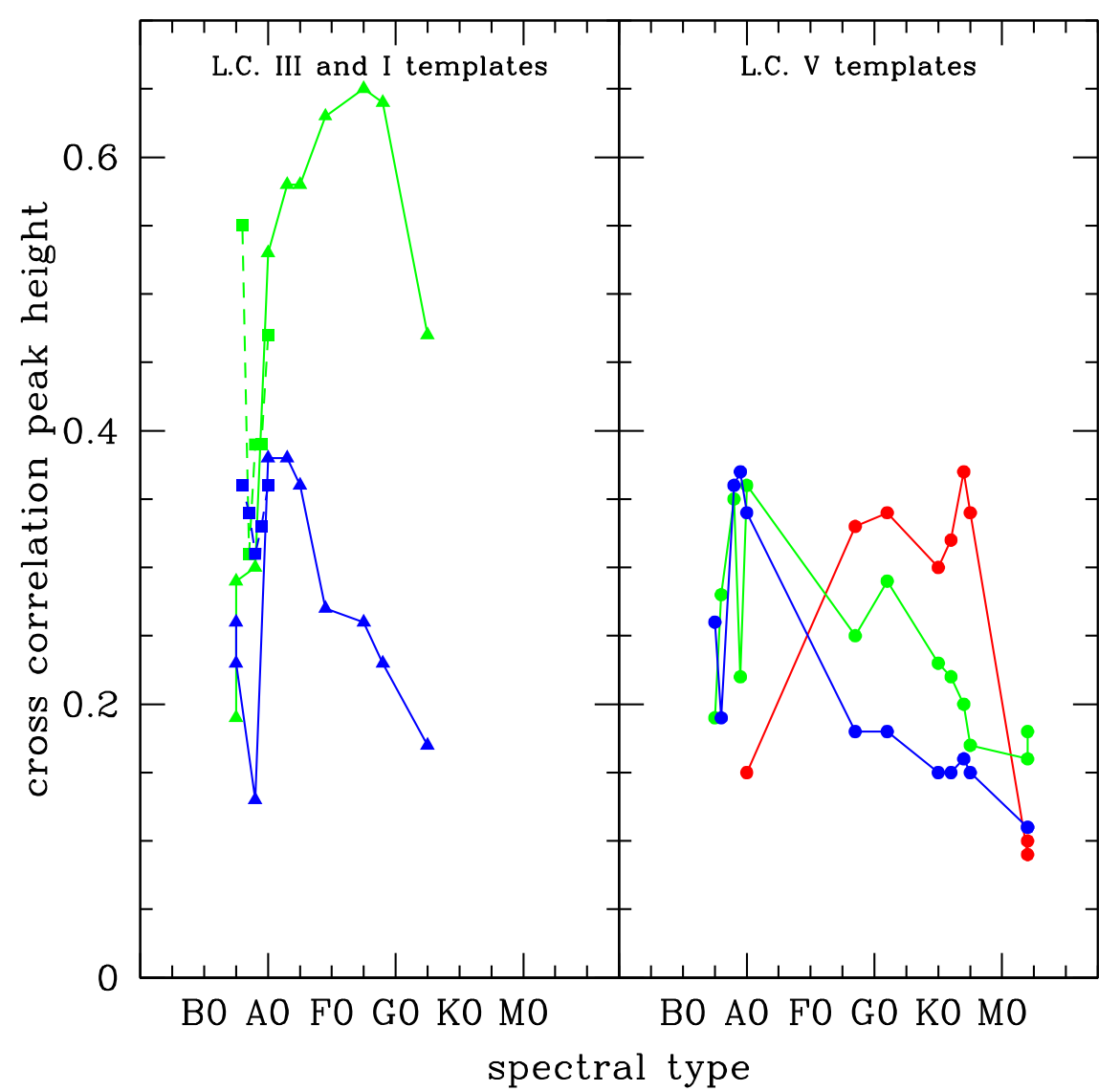

Figure 11. Cross-correlation peak heights as a function of spectral type for low-gravity (left panel) and dwarf (right panel) templates. The analyzed PTF 14jg spectrum is that from 2015 October 27 (shown in Figure 17), taken 625 days after the estimated light-curve peak. Blue points are from the 5200-5285 $\AA$ region, green

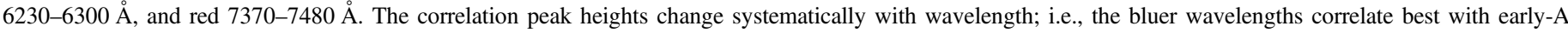
spectral types, while the redder wavelengths correlate best with late-F through $\mathrm{K}$ spectral types. The overall strongest correlations are with supergiants of late-A to lateF spectral type in the "green" part of the spectrum.

the similar excitation potentials, though the lines do not extend to as high a blueshift.

The S II 5606, 5616, 5640, 5645, 5660, 5664, $6386 \AA$ lines are present, though bluer lines of this species are not. At $13.6 \mathrm{eV}$, this is another typical supernova line (seen in Type Ia events but not in core-collapse events).

The $\mathrm{H} \alpha$ line is in absorption, seemingly blueshifted, and appears similar to $\mathrm{O} \mathrm{I}$ in profile, though less deep. The $\mathrm{H} \beta$ is not covered in the first two high-dispersion spectra (but is in our later data); $\mathrm{H} \gamma$ mimics the $\mathrm{H} \alpha$ profile in both shape and strength.

The He I 5876 and $6678 \AA$ lines appear to have blueshifted absorption between -200 and $-500 \mathrm{~km} \mathrm{~s}^{-1}$, though this identification is not strong given the confused nature of the spectrum. The emission component in the $6678 \AA$ profile is likely the Fe I line at the same wavelength.

Summarizing, at low velocities, between 0 and $-150 \mathrm{~km} \mathrm{~s}^{-1}$, there is absorption by $\mathrm{HI}, \mathrm{OI}, \mathrm{Si}$ II, and $\mathrm{Mg}$ II with full line widths extending further to the blue than the photospheric line width, up to $-500 \mathrm{~km} \mathrm{~s}^{-1}$. Over these same 0 to $-150 \mathrm{~km} \mathrm{~s}^{-1}$ velocities, there is broad Ca II triplet and Fe II $5018 \AA$ emission but $\mathrm{P}$ Cygni absorption in these lines at the higher velocities out to -400 to $-500 \mathrm{~km} \mathrm{~s}^{-1}$. Beginning at $-150 \mathrm{~km} \mathrm{~s}^{-1}$ and going to -450 or so, there is a strong multicomponent absorption seen in Ca II H \& K, Ca I $4226 \AA$, Na I D, Li I $6707 \AA$, and likely K I 7665, $7699 \AA$, as well as the continued absorption in the Ca II triplet and Fe II $5018 \AA$. Given the strength and breadth of the $\mathrm{Ca}$ II $\mathrm{H} \& \mathrm{~K}$ and $\mathrm{Na}$ I D lines, the absence of stronger KI 7665, $7699 \AA$ and any Sr II $4077 \AA$ blueshifted absorption seems notable.

\subsection{Prominent Emission Features}

As illustrated in Figure 6, the only strong emission lines in the optical spectrum of PTF $14 \mathrm{jg}$ come from calcium. Both the Ca II 8498, 8542, $8662 \AA$ triplet lines and the [Ca II] 7291 and $7324 \AA$ doublet are present. Their spectral evolution is highlighted in the right panels of Figure 12. These calcium lines are related, where the $7291 \AA$ forbidden line is the ground-state follow-on transition to the permitted $8542 \AA$ deexcitation, while the $7324 \AA$ forbidden line is the ground-state transition that follows the 8498 and $8662 \AA$ de-excitations (Merrill 1943). The permitted triplet emission is quite common in accreting young stars; the forbidden doublet emission is rare, however.

Near the outburst peak and for at least several months after it, the triplet lines were moderately strong $\left(W_{8542}=-12\right.$ to $-15 \AA$ ), and they showed clear classical P Cygni profiles that were visible even in the low-resolution data. However, by the time of the first high-dispersion spectral epoch covering this wavelength range (Figure 13) at 303 days post-peak, the measured $W_{8542}$ was only $-5.5 \AA$, and there was no evidence for $\mathrm{P}$ Cygni structure. The profiles at this later time had $\sim 120 \mathrm{~km} \mathrm{~s}^{-1}$ widths, and the peaks were distinctly blueward of 
line center by about $\sim 40 \mathrm{~km} \mathrm{~s}^{-1}$. The profiles extended to $-200 \mathrm{~km} \mathrm{~s}^{-1}$ on the blue side but were missing their shoulder on the red side. This blue flux asymmetry is reminiscent of magnetospheric accretion-line profile models (Azevedo et al. 2006; Kurosawa \& Romanova 2012 and references therein) in a system having moderate inclination. Königl et al. (2011) argued that such geometries can also be present in highaccretion FU Ori systems, with the magnetosphere located simply closer to the star but not crushed entirely.

As just discussed, the Ca II triplet lines are broad and blueshifted. However, the forbidden [Ca II] 7291 and $7324 \AA$ doublet that follows the triplet lines exhibits narrow lines, with $\sim 25 \mathrm{~km} \mathrm{~s}^{-1}$ FWHM widths (Figure 10). This doublet shares the same radial velocity as the series of weak and narrow permitted emission lines that also appeared in the early spectra of PTF 14jg (discussed in the next section). The moderately strong [Ca II] lines have a clearly Lorentzian line shape and equivalent width $-1.2 \AA$. We note that [Ca II] is the only forbidden line species detected in PTF 14jg. No forbidden emission from the so-called "nebular" lines of [O I], [N II], or [S II] that are quite common in young accretion/outflow systems was obvious at any epoch.

The lack of $\mathrm{H} \alpha$ (Figure 12) is puzzling, as this line is always strongly in emission when strong $\mathrm{Ca}$ II triplet emission is detected in young stars. However, the $\mathrm{H} \alpha$ line in PTF $14 \mathrm{jg}$ is apparent only in shallow weak absorption $\left(W_{\mathrm{H} \alpha} \approx 1.8 \AA\right.$ ), with probable $\mathrm{P}$ Cygni structure or at least a blue-side asymmetry. The line structure could also be explained by contamination from a nearby but unidentified high-excitation species that appears in absorption. Similar blueshifted and very weak absorption profiles may characterize the lines of $\mathrm{He}$ I at 5876, 6678 , and $7065 \AA$ as well in the first few spectral epochs.

Figure 12 also illustrates in the early low-dispersion spectra of PTF 14jg emission from Mg I $8806 \AA$, Fe I $8787 \AA$ (multiplet 60 ), and a few other, even weaker Fe I lines. As the source fades from peak, these lines become weaker, with the $\mathrm{Mg} \mathrm{I}$ $8806 \AA$ line even going into absorption by about 9 months post-peak.

In the infrared (Figure 7), the most prominent emission lines in the early low-dispersion spectra are from $\mathrm{Mg} \mathrm{I}$, with features at 1.1831 (strong), 1.2087, 1.4880, 1.5029 (strong), 1.5044 (strong), 1.5052 (strong), 1.5745, 1.5753, 1.5767, 1.5892, and 1.7111 (strong) $\mu \mathrm{m}$. Weak emission was also detected in various Ca I, Fe I, and possibly Si I lines. Despite the lack of H I Balmer emission associated with the outburst, there is a hint of emission in the H I Brackett gamma line with $W_{21661}=-2.0 \AA$ and $\mathrm{FWHM}=500 \mathrm{~km} \mathrm{~s}^{-1}$; however, problems with telluric correction that might introduce this effect cannot be ruled out. We have only two early-stage spectra of PTF 14jg and have not been able to follow the infrared spectral evolution as we did in the optical.

\subsection{Weak and Narrow Cool Emission Spectrum bear the Outburst Epoch}

The early high-dispersion spectrum, taken only 12 days postpeak, revealed a weak and narrow $\approx 25-30 \mathrm{~km} \mathrm{~s}^{-1}$ FWHM emission-line spectrum superposed on the broad and confused absorption spectrum. Figure 9 illustrates the coincidence of many of these narrow lines with those that are seen more clearly against a flatter continuum in the somewhat extreme young stellar object V1331 Cyg. Figure 10 includes a few of these narrow-line profiles.
The early-stage narrow emission in PTF 14jg is mostly in neutral species, e.g., Fe I, MgI, and $\mathrm{CaI}$, though no $\mathrm{Ti} \mathrm{I}$ is identified, whereas that of V1331 Cyg has both neutral and ionized species. When a narrow metallic emission spectrum is seen in young stars, it is more typically comprised of ionized species such as Fe II and Ti II, not the neutral species.

Correlating the inverted spectrum with a grid of spectral standard stars resulted in consistently good correlation heights and coefficients over a range of temperatures from early-M through early-G types. We take this as an indication that the narrow emission spectrum corresponds to temperatures between $\sim 3700$ and $6000 \mathrm{~K}$.

Weak emission was also detected in the early-stage infrared spectrum with the same Mg I, Ca I, and Fe I species as seen in the optical present in the near-infrared.

The cool gas likely sits above the underlying broad absorption in the photosphere, which comes from a hotter spectral component. As noted above, there is an offset of $(-26$ to -38$)=+11.5 \mathrm{~km} \mathrm{~s}^{-1}$ of the narrow-line emission spectrum with respect to the systemic velocity of the photosphere. One possible scenario is a nearby interstellar cloud, or a cirumstellar cloud located at high latitude toward the pole of the star/disk system, that is radiatively excited by the new influx of photons from the outburst. We mention again that the weak emission spectrum lasted no more than 6 months (based on the second Keck/HIRES spectrum), but at least 6 weeks (based on the second Palomar/TripleSpec spectrum), after the outburst peak.

\subsection{Spectrum Changes during the Fade}

Considering the entire set of spectroscopic follow-up observations, there was some undramatic spectral evolution over the nearly 4 post-peak yr. As the light curve faded by several magnitudes, the optical continuum shape remained essentially the same, consistent with the only modest broadband color changes reported in Section 4.2. There was also little change inferred in the temperature of the absorption spectrum during the early fade, with the high-resolution spectrum essentially unchanged in terms of the absorption species until nearly $2 \mathrm{yr}$ post-peak. At that time, having emerged from the previous wind-dominated hotter absorption spectrum, a cooler absorption component in the spectrum was revealed.

The modest evolution in the profiles of various strong lines is illustrated in Figure 13. Overall, the broad and hot absorption spectrum exhibited only minor morphology changes over time, retaining its general features for the most part. Specifically, as PTF 14jg faded photometrically, some lines became narrower, losing power from their highest-velocity absorption. The line depth closer to line center remained constant, however.

The evolution of the strong outflow lines is shown in Figure 14. Figure 15 demonstrates that the outflow velocities declined sharply as the light curve faded through one $e$-folding time (111 days). The Na I D lines have the shallowest slope, meaning that the maximum velocity did not change appreciably as the source brightness declined, though there is a general decrease in the line depth in nearly all velocity ranges (Figure 14). Several of the distinct components in the blueshifted absorption profile remain steady. The highestvelocity deep components disappear, however. This behavior suggests that much of the $\mathrm{NaI} \mathrm{D}$ absorption comes from stationary locations through which moving gas (ejected in the outburst and decelerating) is flowing. 

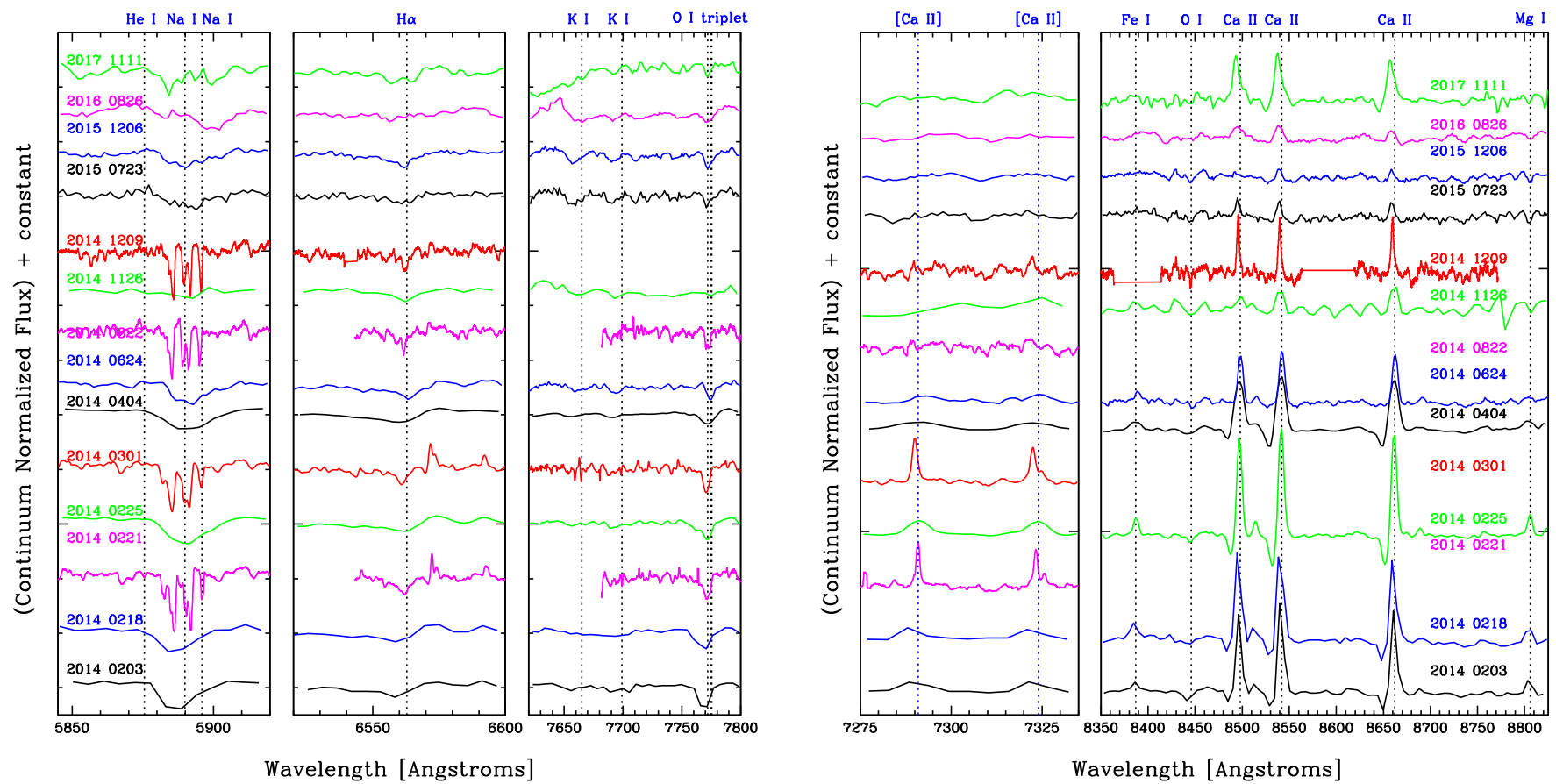

Figure 12. Evolution of spectral features in the PTF 14jg optical spectrum. The left panels show absorption in H $\alpha$ (weak), Na I, K I, and O I. The right panels show prominent emission lines in Ca II and [Ca II]. The resolution varies among the spectra, with those from 2014 February 21, 2014 March 1, 2014 August 22, and 2014 December 9 significantly higher than the others (and smoothed here by a factor of 31). Even accounting for the differences in spectral resolution, the variability over time in the $\mathrm{Ca}$ II and [Ca II] line strengths is real, as is the disappearance of the early-stage Fe I and $\mathrm{Mg}$ I emission.

The P Cygni structure in the Ca II triplet apparent in the early low-dispersion data also disappeared over time (Figure 12), consistent with the weakening wind signature in other lines. In spectra taken more than $\sim 180$ days after the light-curve peak, the emission part of the line was observed to vary in strength with $W_{8542}=-7$ to $-4 \AA$. Unfortunately, this red spectral range was not covered at high dispersion until our third HIRES spectrum, taken $\sim 300$ days after maximum light, by which time both the P Cygni feature of the triplet and the overall weak narrow emission component of the spectrum had disappeared. Figure 13 illustrates strength and morphology changes in the (blueshifted) emission part of the line during the epochs of high-dispersion coverage. However, a later low-dispersion spectrum-taken almost $4 \mathrm{yr}$ after the initial rise-once again had P Cygni structure in the Ca II triplet (see Figure 12), with emission strength comparable to the early burst stage. We have no contemporaneous photometric monitoring of the source at this late time.

The narrow emission spectrum in neutral species such as Fe I, Mg I, and Ca I was present only in the first high-dispersion spectrum, taken within 2 weeks of maximum light, and had disappeared by the next high-dispersion spectrum 6 months later. The [Ca II] doublet emission weakened over the same time period.

The stronger Fe I $8787 \AA$ and Mg I $8806 \AA$ that were visible in the early low-dispersion data also gradually weakened, with the Mg I line actually evolving to appear in absorption some time after 4.5 months post-peak (see rightmost panel of Figure 12).

Returning to the absorption spectrum, there was evidence by $\sim 300$ days post-peak for development of a narrower neutral absorption component, specifically in Fe I and Mg I (e.g., 4481 and $6318 \AA$ ). By 625 days post-peak, signatures of a K-type absorption spectrum began to emerge in the high-dispersion data (see Figure 17 below for illustration). In addition, we call attention to the clear presence of Li I $6707 \AA$ in PTF 14jg at this late time.

By nearly $4 \mathrm{yr}$ after the outburst, in late 2017, the lowresoluton absorption spectrum of PTF 14jg did evolve somewhat, with significant weakening of Si II 6347, $6371 \AA$ and the nearby $\mathrm{Mg}$ II absorption (see Figure 6). This may indicate further continued cooling of the wind-launching region. The composite spectrum nature of the PTF 14jg absorption spectrum was maintained over this time period, as illustrated in the cross-correlation analysis of Figure 11.

\section{Synthesis of Evidence to Date: A Possible FU Ori Star}

In this section, we discuss PTF $14 \mathrm{jg}$ as a likely young star outburst. Given its location in the Galactic plane, projection near a known star-forming region, and the evidence for ultraviolet/infrared excess, as well as Li I, this is a reasonable hypothesis to explore. Furthermore, the observed properties of the PTF 14jg outburst are not a good match to other categories of plausible outburst objects, as discussed in Section 9. We believe the source to be a likely FU Ori star, though of larger amplitude and hotter than those members of the class identified heretofore.

\subsection{Location and Environment}

PTF 14jg is projected on the sky about a degree away from the young massive star cluster IC 1805 and outside the H II region. In a similar fashion, FU Ori itself is well north and west of the active star-forming clouds in Orion. And both V1515 Cyg and V1057 Cyg are likewise located away from the molecular clouds assocated with Cyg X and the North America Nebula, respectively. The recently discovered bona fide FU Ori star 2MASS J06593158-0405277 (V960 Mon) is also several 


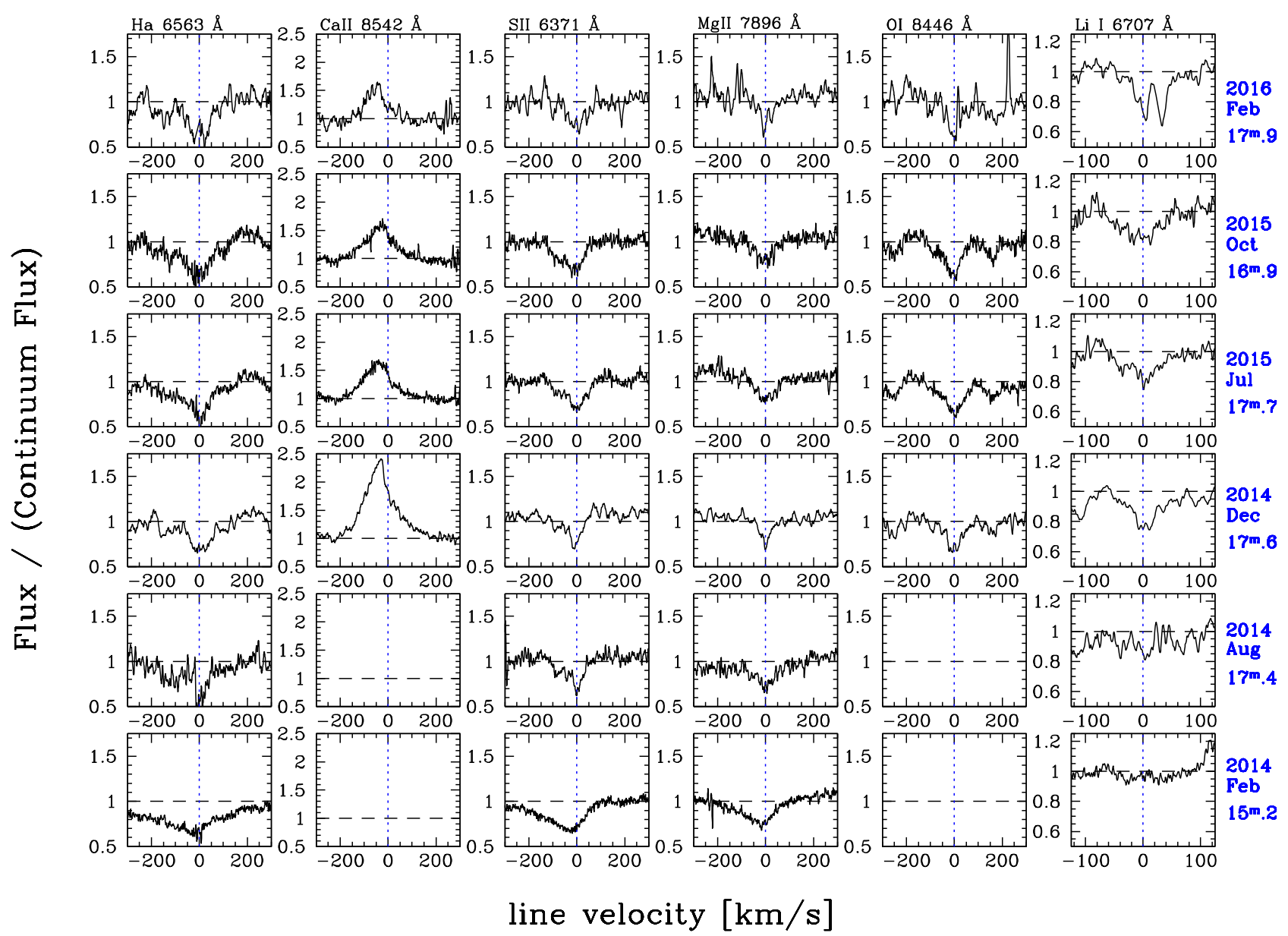

Figure 13. Evolution of permitted line profiles. Spectra are sequenced from bottom to top with source magnitudes given at right. The velocity scale is $\pm 300 \mathrm{~km} \mathrm{~s} \mathrm{~s}^{-1}$ for all panels except the rightmost, which is $\pm 125 \mathrm{~km} \mathrm{~s}^{-1}$. A velocity shift of $38.1 \mathrm{~km} \mathrm{~s}^{-1}$ has been applied to correct for the systemic radial velocity inferred from the late-epoch absorption-line spectrum. Narrow core $\mathrm{H} \alpha$ emission may have developed in the most recent spectrum, though the Ca II triplet lines are still broad and have an asymmetric profile that is peaked at negative velocity. Blueshifted velocities in Si II and Mg II have decreased as the source has faded. The Fe II and O I profiles are essentially unchanged.

degrees north of the well-studied star-forming regions in Canis Majoris, with which it has been associated. It is perhaps more the rule than the exception that FU Ori eruptions are found outside the main active regions of star formation. However, given that most FU Ori events were identified at optical wavelengths, these locations could reflect a bias toward events associated with less obscured, and perhaps thus older, young stellar objects. A contrasting case is that of PTF 10qpf/HBC 722, which is in the heart of the main embedded cluster associated with the North America Nebula region.

In this general direction on the sky, only the mainly local clouds at $v_{\mathrm{LSR}}=0 \mathrm{~km} \mathrm{~s}^{-1}$ and the $2 \mathrm{kpc}$ Perseus arm at $v_{\mathrm{LSR}}=-40 \mathrm{~km} \mathrm{~s}^{-1}$ are seen in $\mathrm{CO}$ emission, with little beyond. The $-38.1 \pm 1.2 \mathrm{~km} \mathrm{~s}^{-1}$ heliocentric velocity of PTF $14 \mathrm{jg}$ corresponds to a $v_{\mathrm{LSR}}$ of $-34.7 \mathrm{~km} \mathrm{~s}^{-1}$, which would place the object on the near side of the $2 \mathrm{kpc}$ arm. Indeed, Georgelin \& Georgelin (1970) measured $\mathrm{H} \alpha$ velocities around the nearby $\mathrm{H}$ II regions, reporting $v_{\mathrm{LSR}}=-36.6 \pm 2.6 \mathrm{~km} \mathrm{~s}^{-1}$.

There is an outer spiral arm beyond the Perseus arm, located at $6 \mathrm{kpc}$ and $v_{\mathrm{LSR}}=-100 \mathrm{~km} \mathrm{~s}^{-1}$. Although this arm is not apparent at the exact position of PTF 14jg, it is seen about $10^{\circ}$ closer to the inner Galaxy (Heyer et al. 1998). At the exact position of PTF 14jg, there is no clear $\mathrm{CO}$ emission at any velocity. The cloud catalog of Heyer et al. (2001) shows the closest cloud in projection to be HC 3927, which is located between $l=135.92-136.03$ and $b=0.70-0.84$ at a velocity of $-75.92 \mathrm{~km} \mathrm{~s}^{-1}$. Its centroid is about $9 ! 3$ away from PTF 14jg, corresponding at the kinematic distance of $9.71 \mathrm{kpc}$ to $26 \mathrm{pc}$. The radial velocity of PTF $14 \mathrm{jg}$ is not consistent with these larger distances or anything much further than about $2 \mathrm{kpc}$.

Examination of 2MASS $\left(J H K_{s}\right)$ and WISE $(W 1, W 2, W 3$, W4) infrared colors of stars in a $15^{\prime} \times 15^{\prime}$ box shows a general similarity in various color-color diagrams between a field centered on PTF 14jg and a field centered within the nearby $\mathrm{H}$ II region (but south of the IC 1805 cluster). There is no dominant population of infrared excess sources at either location.

\subsection{Light Curve}

The well-characterized brightening of PTF 14jg is large in amplitude, $>6$ mag in the red optical, and $>4$ mag in the nearinfrared. For comparison, the recent PTF 10qpf event that was also captured by PTF (Miller et al. 2011; see also Semkov et al. 2010) in the star LkH $\alpha 188$ G4 (also known as HBC 722 and now as V2493 Cyg) was smaller, at about $4 \mathrm{mag}$ in the red optical and 3 mag in the near-infrared. The classical events FU Ori and V1057 Cyg were observed only in the blue optical, and both had amplitudes around $5.5 \mathrm{mag}$ (Herbig 1977). 


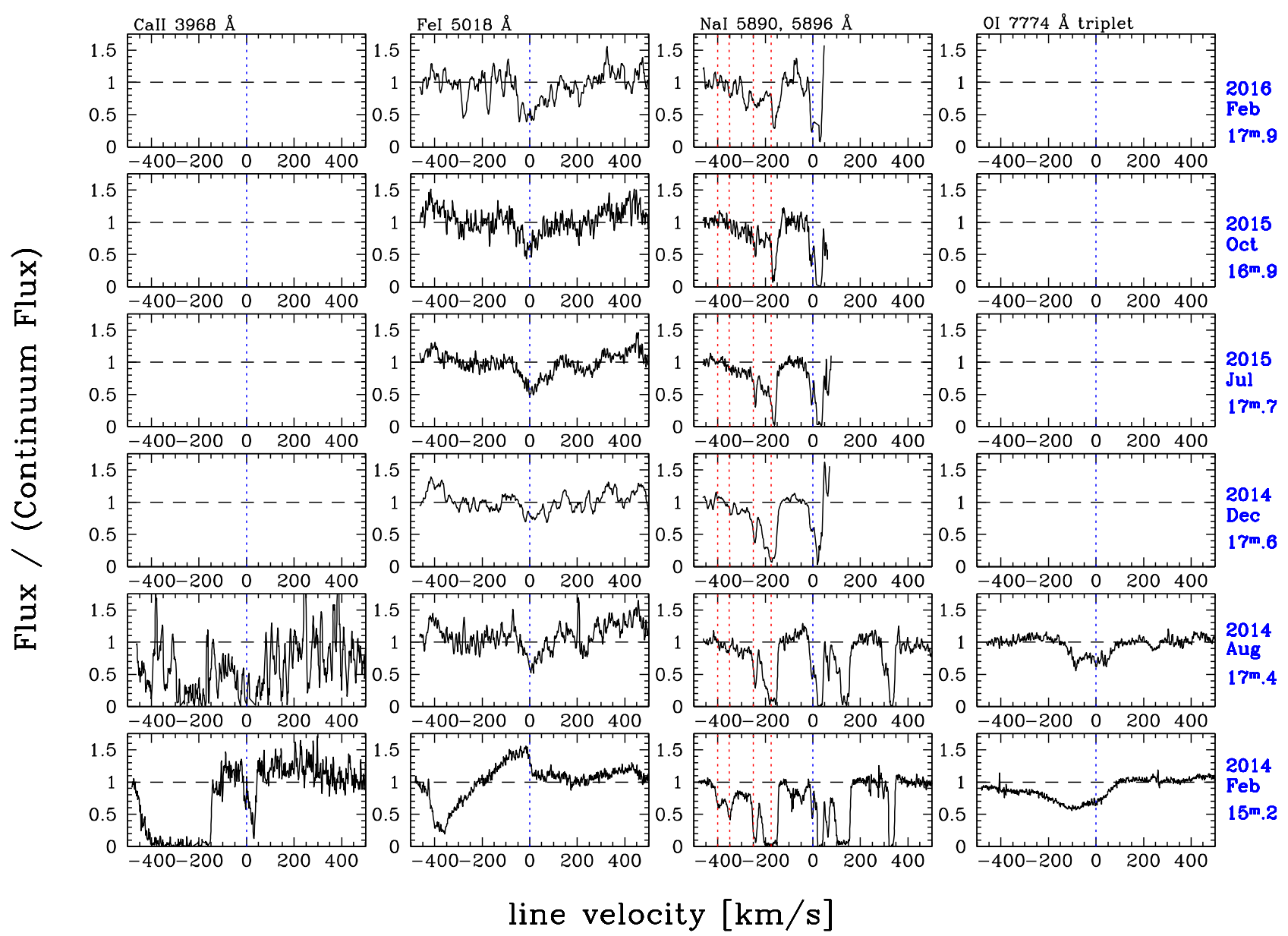

Figure 14. Evolution of wind-dominated line profiles. Spectra are ordered as in Figure 13, with the same adopted systemic velocity. Here the velocity scale is $\pm 500 \mathrm{~km} \mathrm{~s}^{-1}$ for all panels. Red vertical lines in the Na I D2 panels indicate semi-stable absorption components at $-175,-250,-350$, and $-400 \mathrm{~km} \mathrm{~s}{ }^{-1}$. The two additional features at $\sim-50$ and $-100 \mathrm{~km} \mathrm{~s}^{-1}$ are actually the -400 and $-350 \mathrm{~km} \mathrm{~s}^{-1}$ components of the Na I D1 line. The Ca II $\mathrm{K}$ (\& $\mathrm{H}$ ) lines exhibit continuous saturated absorption over this entire velocity range, extending at the terminus to $\sim-530 \mathrm{~km} \mathrm{~s}^{-1}$.

The outburst of PTF $14 \mathrm{jg}$ is much larger $(6.7 \mathrm{mag}$, according to our sigmoid fit) and has lasted much longer (nearing $5 \mathrm{yr}$ at the time of this writing) than the temporary brightness increases associated with EX Lup-type burst events; these are also accretion-driven brightenings of young stars, typically only 1-2 mag in amplitude and only months- to year-long in duration.

During its few-month rise, the form of the light curve of PTF 14jg was concave during approach to peak brightness. This is consistent in terms of both timescale and shape with other FU Ori stars. In Figure 16, we compare the early lightcurve behavior among the recent and well-documented outbursts of PTF 14jg and PTF 10qpf (HBC 722) and show the available historical data for the outburst of the prototype $\mathrm{FU}$ Ori and the later V1057 Cyg. The rise time of PTF 14jg is quite similar to that of PTF 10qpf (HBC 722), as well as that of V960 Mon (not shown), and comparable to that of FU Ori, though shorter than that for V1057 Cyg. The other canonical FU Oritype star, V1515 Cyg, had a much slower rise and a less welldefined "peak," as well as a much lower amplitude.

The timescale of the PTF 14jg rise, approximately 112 days, can be related to a size scale assuming the thermal diffusion timescale (that appropriate for the instability to propogate from its origin to the star) is approximately the sound-crossing timescale, $r / c_{s}$. Using standard equations for an accretion disk and assuming typical classical $\mathrm{T}$ Tauri parameters leads to $\tau_{\text {thermal }} /$ day $=\left(r / R_{\odot}\right)^{11 / 8}\left(M / M_{\odot}\right)^{-1 / 8}$. Estimating for the PTF 14jg progenitor that $R_{*} \approx R_{\odot}$ and $M_{*} \approx M_{\odot}$ (each accurate to within a factor of two, assuming a young premain-sequence star), the rise time suggests a location of $\sim 32 R_{\odot}$ or 0.15 au for the outburst. The true thermal diffusion timescale will be larger than $r / c_{s}$ and requires adopting a value of $\alpha$. Even the approximate location suggests that the outburst is driven by an inner disk instability mechanism rather than a magnetospheric event.

The detailed behavior of the FU Ori star brightness declines from maximum is diverse (Figure 16). The morphology of the immediate post-peak light curve of PTF 14jg does share some similarities with other FU Ori stars. Like PTF 14jg, PTF 10qpf (HBC 722) exhibited an immediate fade but then a smooth and slow rebrightening. In contrast, the implied rebrightening in PTF 14 jg after $\approx 400$ days and the detected rebrightening and tertiary local maximum after $\approx 600$ days were both more abrupt. See the figures in Clarke et al. (2005) for further comparison with early-stage behavior in the light curves of V1057 Cyg and FU Ori.

Over time, however, the PTF 14jg light curve became more consistent with an exponential shape. As described in Section 6 , the measured $e$-folding time is 111 days $(0.3 \mathrm{yr})$, with the photometric variation about the fitted exponential only 0.09 mag (rms). Several other recent FU Ori stars with highquality outburst data, such as PTF 10qpf (HBC 722) and V960 


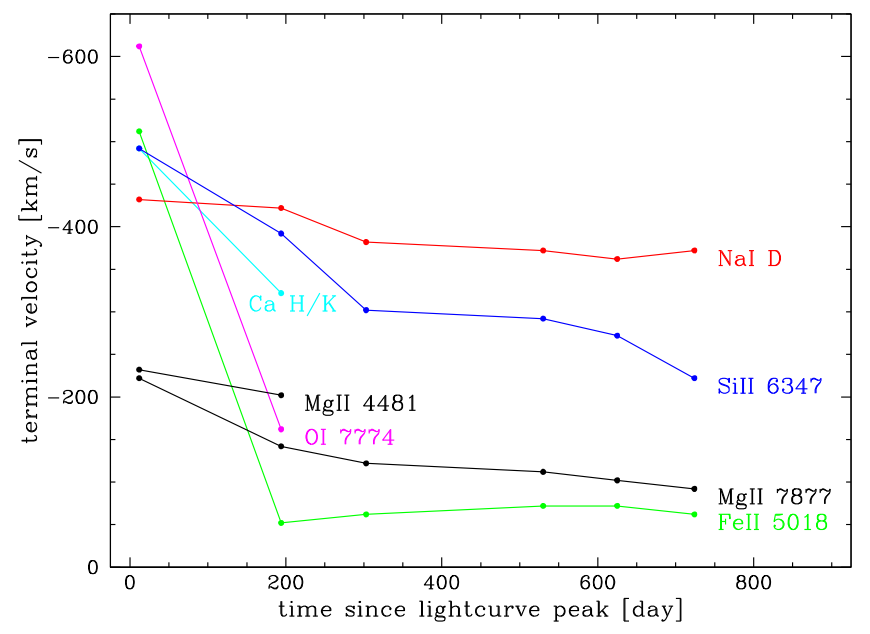

Figure 15. Evolution of blue-side terminal velocities for absorption lines with wind signatures in Figures 13 and 14. Velocities are in the rest frame of the source and have estimated measurement errors of $10-20 \mathrm{~km} \mathrm{~s}^{-1}$. The wind speeds were highest at outburst, then declined at different rates for different lines, mostly within the first one to two $e$-folding times of the light curve. The $\mathrm{Na}$ I D lines exhibited the least change. The O I measurement is overestimated due to the triplet nature of the line.

Mon, also exhibit little scatter in the outburst photometry. In contrast, lower-amplitude, shorter-lived outbursts in objects like EX Lup and V1647 Ori show variability at the $\approx 0.5-1$ mag level in their outburst phases.

Notably, an exponential decline also fits the post-peak light curve of V1057 Cyg, the most rapidly declining classical FU Ori-type star, but with a much longer $e$-folding time of 2307 days $(6.3 \mathrm{yr})$ derived utilizing the data published in Clarke et al. (2005). The post-peak times $t_{1}, t_{2}$, and $t_{3}$ for V1057 Cyg are $\sim 650,1950$, and 4400 days-factors of $\sim 10,10$, and 5 longer than those of PTF 14jg (54, 139, and 747 days, as described in Section 6). Notably, Kraus et al. (2016) reported that the long-accepted FU Ori object V346 Nor had decayed on a timescale of just a few decades, rather than the nominal century that is typically assumed for FU Ori event cooling. Although there are substantial timescale differences among members of the FU Ori class, the light curve of PTF 14jg is most similar to that of V1057 Cyg in terms of the basic exponential profile of the decline.

In addition to its similarity to the FU Ori star V1057 Cyg, the exponential decline in the PTF 14jg light curve resembles that of classical novae. However, the timescale of the PTF 14jg fade is much longer than that of novae. Nova outbursts are caused by thermonuclear runaway and detonation, rather than by instability in an accretion disk. Type Ia (luminous) supernovae also exhibit exponential fades. In these objects, the decline is mapped onto the radioactive decay of particular isotopes following the explosion. Destructive explosion scenarios fail overall for PTF 14jg in several ways (see Section 9).

In terms of color comparisons, only the recent PTF 10qpf (HBC 722) has been observed well enough for definitive statements. Semkov et al. (2017) showed that the burst itself was a blueing event, with $V-I$ color decreasing by $\sim 1 \mathrm{mag}$, then increasing in the immediate post-peak period by $\sim 0.5 \mathrm{mag}$ before again becoming slightly bluer after about $1 \mathrm{yr}$, and finally settling around 0.5 mag bluer than the pre-burst colors. For PTF 14jg, there is no pre-burst optical color information, but the source clearly has very blue ultraviolet and optical colors in the immediate post-peak period. Unlike PTF 10qpf, the initial fade of PTF 14jg is relatively colorless in the optical but does redden slightly after a few months (Figure 4).

\subsection{Outburst Luminosity}

The luminosity of PTF 14jg can be estimated from the peak magnitude of $R_{\mathrm{PTF}}=14.93$, an assumed distance, and an extinction estimate.

Our radial velocity measurement appears to confirm the association with the nearby $W 3 / W 4 / W 5$ complex at $1.95 \mathrm{kpc}$. However, as noted above, while this is the only known region of recent star formation anywhere close to the direct line of sight, there is also a further spiral arm beyond the Peseus arm in this general direction that is not detected at the exact location of PTF 14jg but can also be considered as a plausible distance.

Using the extinction derived from the SED fitting of $A_{V}=4.75$ or $A_{R}=3.56 \mathrm{mag}$ and adopting the bolometric correction appropriate to an A0 star (about -0.25 mag), a source located at 2 or $6 \mathrm{kpc}$ would have a luminosity of 114 or $1014 L_{\odot}$. Changing the dominant spectral type would alter the bolometric correction by only a few tenths. Simple scaling to solar values also yields $100 L_{\odot}$ for the preferred $2 \mathrm{kpc}$ distance. The luminosity can also be estimated by integrating the limited range that we have sampled photometrically near the peak of the SED. Fitting a model that accounts for extinction (see Figure 8) yields $\sim 130 L_{\odot}$, modulo the exact temperature and extinction used in the fit. Typical FU Ori luminosities are several hundred $L_{\odot}$, though V900 Mon is also only $\sim 100 L_{\odot}$, and PTF 10qpf (HBC 722) is a notable outlier at only $\sim 12 L_{\odot}$. The recently announced Gaia 17bpi (Hillenbrand et al. 2018) is even lower at only $\sim 8 L_{\odot}$.

We believe that the large luminosity of PTF $14 \mathrm{jg}$ is produced in an accretion disk. However, if instead of a disk, the luminosity is (hypothetically) generated by a normal stellar photosphere, then assuming 114 (or 1014) $L_{\odot}$ and the $9700 \mathrm{~K}$ temperature corresponding to the A 0 spectral type, a radius of 3.8 (11.3) $R_{\odot}$ is implied for the PTF 14jg photosphere. The location in the H-R diagram of such a source would be on (near) the main sequence, implying a mass of 3 (5) $M_{\odot}$. This radius would also be consistent with both the large rotation rate implied by the $100-150 \mathrm{~km} \mathrm{~s}^{-1}$ line broadening that we observe and the high terminal velocity seen in the outflow, presuming this is also the escape velocity from the star. For the supergiant classification of the spectrum, instead of the $10^{2-3}$ $L_{\odot}$ and 3-5 $M_{\odot}$ calculated above, such an object should (Abt \& Morrell 1995; Verdugo et al. 1999) have $10^{4-6} L_{\odot}$ and $10-40 M_{\odot}$, with a much larger radius $\left(30-100 R_{\odot}\right)$ as well as slower rotation $\left(20-40 \mathrm{~km} \mathrm{~s}^{-1}\right)$ and smaller escape velocity $\left(<275 \mathrm{~km} \mathrm{~s}^{-1}\right)$. Another factor of several in distance-beyond the $6 \mathrm{kpc}$ arm-is required to get above $10^{4} L_{\odot}$ and into the lower-luminosity regime of normal A0 supergiants, or roughly a factor of 10 in distance to come close to the $10^{5} L_{\odot}$ realm more typical of early-A supergiants.

The lack of consistency of the above logic lends credence to an accretion disk origin for the high source luminosity. The spectrum of PTF $14 \mathrm{jg}$ is very clearly dominated by a hot component, but the photosphere also has low surface gravity. Yet no single-temperature "normal" stellar source can fit all of the observational constraints. 


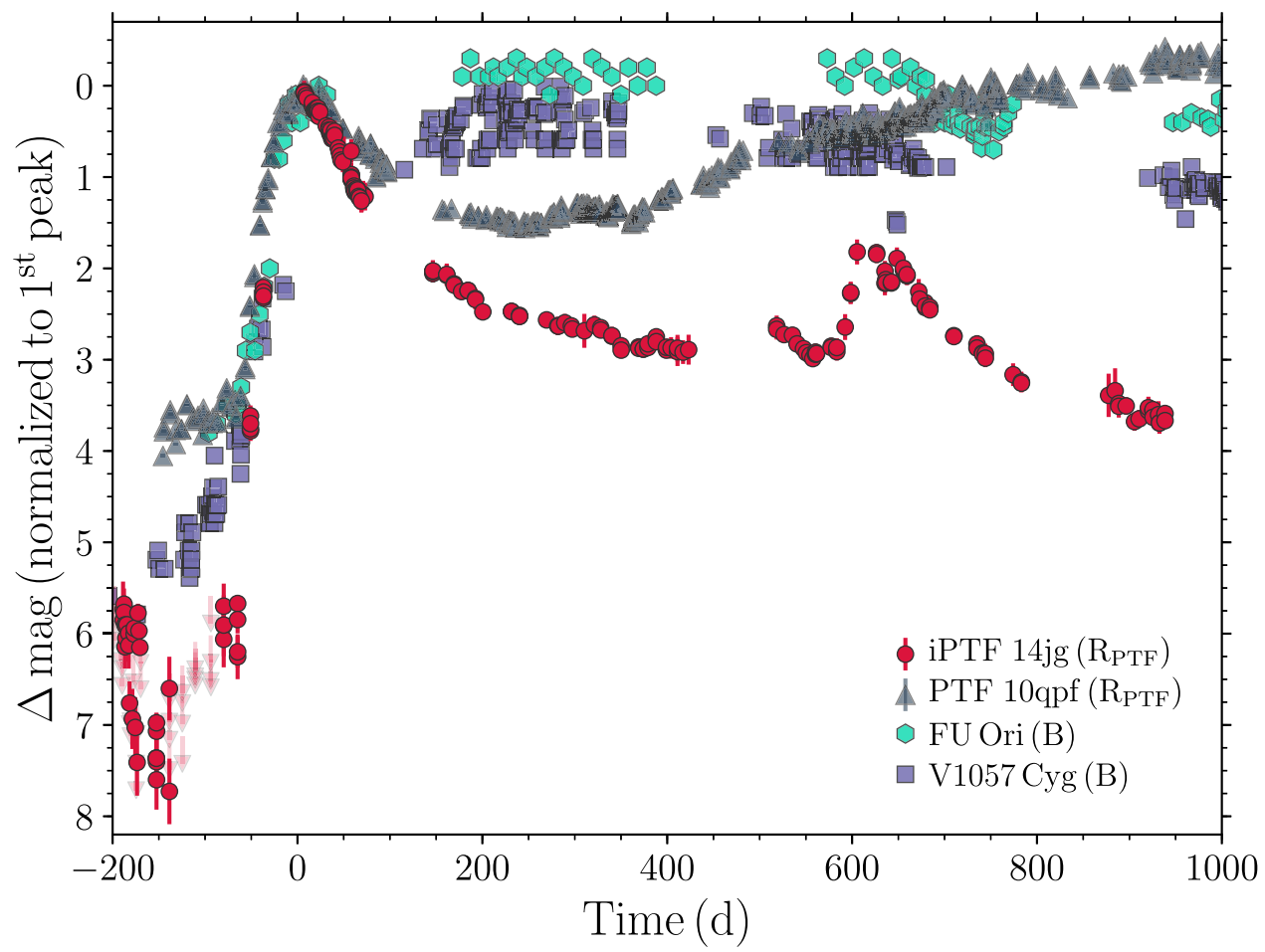

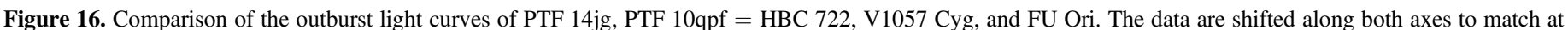

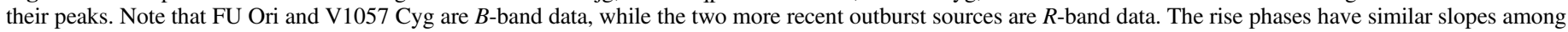
the objects, albeit different amplitudes. The post-peak light-curve shapes are very diverse.

\subsection{Absorption Spectrum}

The PTF 14jg infrared spectrum is pure continuum, lacking $\mathrm{HI}$ lines as in the optical spectrum but also the characteristic $\mathrm{CO}$ absorption that is a hallmark of FU Ori stars. This is consistent with a hot photosphere. ${ }^{11}$

The optical low-dispersion spectrum of PTF 14jg (Figure 6) initially appeared to be a reasonable FU Ori match, displaying many lines that are seen in FU Ori stars and having similarity to an FG giant or supergiant spectrum but with the notable addition of strong Si II, Mg II, and Fe II absorption that is characteristic of late-B and early-A stars but not FG stars. At high dispersion, besides atypically hot species, the absorption lines exhibited by PTF 14jg are also atypically strong, with EW typically exceeding $1 \AA$. In the redder parts of the optical spectrum, the lines reach $87 \%-90 \%$ depth instead of the $92 \%-$ $98 \%$ depth seen in other FU Ori stars. In addition, at FWHM $\sim 100-150 \mathrm{~km} \mathrm{~s}^{-1}$, the early absorption spectrum was broader than that of all other known FU Ori stars. The hot (highexcitation) absorption lines are likely coming from above the stellar surface, perhaps the inner wind.

We note that most other FU Ori stars have been observed at high dispersion only at much later stages in their outbursts. Even the recent PTF 10qpf (HBC 722) and V960 Mon were sampled at high dispersion for the first time many weeks to months after their outburst peaks. Indeed, PTF 14jg exhibited significant spectral evolution from Figure 9, taken just 12 days post-peak.

Figure 17 shows a comparison of the late-time highdispersion spectrum of PTF 14jg, taken $1.7 \mathrm{yr}$ post-peak, with

\footnotetext{
11 The FU Ori + Herbig Ae/Be binary ZCMa also has no CO absorption in composite spectra, but when spatially resolved as in Hinkley et al. (2013), the absorption in the B-component FU Ori source is apparent.
}

V1515 Cyg. The match is quite good but not exact. Most of the absorption that is present in PTF 14jg but not V1515 Cyg is due to the high-excitation Si II, Mg II, and Fe II mentioned above. Also, PTF 14jg has more highly blueshifted Na I D than V1515 Cyg. Notably, V1515 Cyg is the least line-broadened member of the FU Ori class and is interpreted as having lower inclination than V1057 Cyg and FU Ori, which have much broader absorption lines. The approximate match of widths in the nonwind absorption lines may suggest that PTF 14jg is seen at a low-inclination viewing angle. However, the Ca II triplet line morphology (Figure 13) seemed to suggest a higher viewing angle.

Also apparent in Figures 13 and 17 is that, although there was a lack of clear and strong Li I in the earliest data on PTF 14jg, as the light curve faded, a narrow Li I component did emerge. The early-stage Li I profiles that prevented us from making a much earlier conclusion regarding the young star nature of this object were likely dominated by the outflow. We note that several other accepted FU Ori stars also have ambiguous Li I profiles (e.g., V1057 Cyg) that may be similarly affected by outflow. At early times, the Li I profile seems similar to that of Par 21 or V883 Ori, while at late times, the double-peaked Li I line is reminiscent of profiles exhibited by FU Ori, V582 Aur, and PTF 10qpf/HBC 722.

Finally, we recall that absorption at $6614 \AA$ is present in PTF 14jg. This line is prominent in all FU Ori spectra and is seen in FGK supergiant spectra but not in the higher-gravity giant or dwarf objects. While it seems promising as a gravity constraint, this wavelength is also identified with a diffuse interstellar band (DIB) and is therefore more likely to be seen in more luminous sources. Several other DIBs are also seen in the PTF 14jg spectrum (see Figure 9) with narrower profiles at $\approx 40 \mathrm{~km} \mathrm{~s}^{-1}$, compared to the generally broad absorption 
features. Strong narrow atomic interstellar absorption is also identifiable in the $\mathrm{Ca}$ I, Na I, and KI profiles, whose outflow and zero-velocity components were discussed above.

\subsection{Wind/Outflow Signatures}

As discussed in detail in Section 7.4, PTF 14jg exhibited a wind-dominated optical spectrum over the first 6 months of the outburst, the signatures of which gradually decreased in strength over the subsequent several years, as the source faded photometrically. The wind was shown to exhibit different absorbing components over a wide range of velocities, presumably dictated by the density and temperature in the surrounding medium that allows different lines to form at different distances from the star.

For example, the optical light is coming through a region that is clearly optically thick in Na I D and Ca II H \& K. Both features show an inner edge to the absorption at $-100 \mathrm{~km} \mathrm{~s}^{-1}$ (Figure 14), yet this region between the rest velocity and $-100 \mathrm{~km} \mathrm{~s}^{-1}$ is emitting in the Ca II triplet and - early on-the Fe II $5018 \AA$ line. Hotter lines such as Si II show continuous absorption from the rest velocity out to a terminal velocity that is similar to the $\mathrm{NaI} \mathrm{D}$ and $\mathrm{Ca}$ II $\mathrm{H} \& \mathrm{~K}$ terminal velocity (Figure 15). Likewise, Mg II exhibits continuous absorption from the rest velocity but reaches a much lower terminal velocity.

The standard scenario for radiatively driven outflow is governed by either the Eddington factor $\left(2 \times 10^{-5} L / M\right.$, in solar units) under the most basic considerations or, for linedriven radiative winds, a relation between the wind momentum $\left(\dot{M} v_{\infty}\right)$ and the stellar parameters $\left(\sqrt{L^{3} / R}\right.$ in simplified form). As noted above, the luminosity of PTF $14 \mathrm{jg}$ seems too low for radiative processes to be important in producing the observed outflow. Furthermore, the wind terminal velocity of around $500 \mathrm{~km} \mathrm{~s}^{-1}$ for PTF 14jg is higher by a factor of two than those typical of A supergiants, albeit in the range of values observed for mid-B supergiants (e.g., Kudritzki et al. 1999).

In the FU Ori scenario, the outflow is accretion-driven and emanates from the inner disk region. It then propagates through the dense circumstellar and nearby interstellar medium.

Comparing to the low-dispersion spectra of FU Ori stars, the obvious P Cygni structure in the Ca II triplet emission of PTF 14jg (Figure 12) is consistent with those objects, indicating strong outflow. Notable is the rapid evolution from early epochs to disappearance of the blueshifted absorption by about 4.5 months post-peak in favor of a purely blueshifted emission spectrum in Ca II $8542 \AA$ (Figure 13). Rapid evolution of the line structure was also observed in the early stages of the PTF 10qpf outburst, with the red emission side of the $8542 \AA$ profile decreasing significantly in strength over the first few years, while the blueshifted absorption remained present. The late-time morphology of the Ca II $8542 \AA$ feature in PTF $14 \mathrm{jg}$ is perhaps most similar to V1735 Cyg.

Despite the strong wind signature in Ca II, there was never strong emission or clear $\mathrm{P}$ Cygni absorption in $\mathrm{H} \alpha$ from PTF 14jg. Among FU Ori stars, the $\mathrm{H} \alpha$ profile of PTF 14jg seems most similar to that of V883 Ori and possibly Par 21. It also resembles the higher Balmer lines in PTF 10qpf that had more broadly blueshifted absorption than exhibited in the $\mathrm{H} \alpha$ profile (Miller et al. 2011).

The overall influence of the outflow on line-absorption profiles in PTF 14jg is much more complicated than in the established FU Ori stars. The strongest and hottest outflow signatures are in PTF14jg. For example, we are not aware of any other FU Ori stars that exhibit any hint of an Si II feature. This line has been documented in a few other young stars; e.g., Grinin et al. (2001) illustrated its presence in UX Ori but did not discuss the line. It is also generally present in early F III stars.

The most comparable object may be V1057 Cyg. Like PTF 14jg, V1057 Cyg has a broad, nearly saturated multicomponent outflow in $\mathrm{Na}$ I D with the wind also visible in the K I $7699 \AA$ line. In both PTF 14jg and V1057, the Li I profile has a zero-velocity narrow emission component, along with blueshifted absorption out to about $-100 \mathrm{~km} \mathrm{~s}^{-1}$; the phenomenon is discussed in detail in Herbig (2009) for V1057 Cyg. Notably, the high-excitation Si II lines that are broad and blueshifted in PTF 14jg are also weakly seen at zero velocity in V1057 Cyg, as well as in early spectra of V960 Mon (though weakening significantly a year post-peak in that object).

\subsection{Early-time Emission-line Spectrum}

In the FU Ori interpretation, narrow permitted emission lines at an early stage of the outburst could originate in a region of high density along the poles that is newly illuminated by the hot outburst. Another location for the narrow emission lines could be in the magnetosphere, if the large increase in accretion rate is slow enough that normal magnetospheric emission continues for several weeks or months. As noted above, there is a slight redshift of the narrow emission spectrum by about $11.5 \mathrm{~km} \mathrm{~s}^{-1}$ relative to the inferred systemic velocity from the late-time absorption-line spectrum. If the outburst accretion rate is low enough, the magnetosphere could be maintained instead of crushed. Only weak lines would be seen as narrow emission, while the strong lines would have their narrow magnetospheric emission swamped by the wind signatures, consistent with our observations.

Unusual for FU Ori stars is the presence of forbidden emission at [Ca II] 7291, $7324 \AA$ in PTF 14jg. This is a highdensity line relative to the usual suite of "nebular" forbidden lines. Although not too common in young stars, ${ }^{12}$ this line is weakly present in extreme emission-line objects like V1331 Cyg, V2492 Cyg (PTF 10nvg), and RW Aur, all of which have ubiquitous permitted and forbidden line emission. None of the bona fide FU Ori stars show any forbidden-line emission ${ }^{13}$ (or the He I that characterizes many of the more regularly accreting $\mathrm{T}$ Tauri stars). This means that the FU Ori winds must be more dense than the typical $\mathrm{T}$ Tauri star winds thought to originate near the magnetospheric region.

Hartigan et al. (2004) discussed the [Ca II] doublet in young stars in detail, reporting that the critical density is $5 \times 10^{7} \mathrm{~cm}^{-3}$ and the expected equilibrium ratio of $7291 \AA$ : $7324 \AA=1.5$, relatively independent of density. This is roughly consistent with the measured ratio of $\sim 1.2$ in PTF 14jg. As also noted earlier, all of the Ca II lines that we observe in PTF 14jg are related. The H \& K doublet (which we see in strong and broadly blueshifted absorption) has an upper level that is also the "infrared" triplet line (having classical P Cygni structure) upper level, with its lower level then the forbidden doublet's (narrow emission) upper level. The forbidden doublet's lower level (the ground state) is in

\footnotetext{
${ }^{12}$ It can also be seen in some high-luminosity blue or yellow supergiants that may be post-RSG or post-AGB stars.

13 Exceptionally, V1057 Cyg does exhibit very weak [O I].
} 

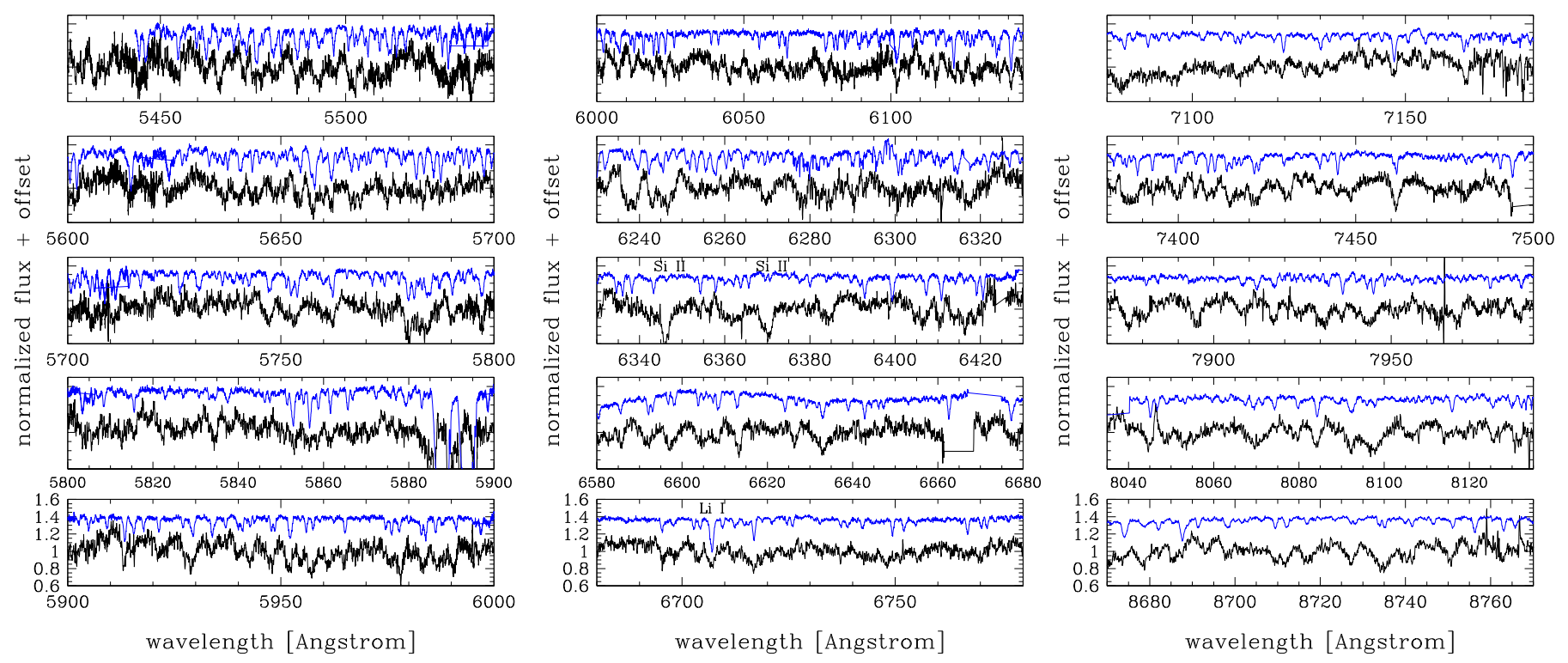

Figure 17. Comparison of the PTF 14jg Keck/HIRES spectrum (in black) from 2015 October 27, when the source was 625 days post-peak, to that of V1515 Cyg (in blue), a bona fide FU Ori star. The normalization is nonoptimal in some orders, and PTF 14jg is clearly broader, with many mainly high-excitation lines still present (most notably Si II at, e.g., 6347, $6371 \AA$, as well as Mg II and Fe II). Nevertheless, the spectral match is reasonable, including the now-clear Li I $6707 \AA \AA^{\circ}$ signature in PTF 14jg. This post-burst evolution of the optical absorption spectrum indicates the development of a cooler photosphere relative to that displayed in Figure 9, from just 12 days post-peak.

common with the $\mathrm{H} \& \mathrm{~K}$ lower level. The deep absorption that we see in the $\mathrm{H} \& \mathrm{~K}$ lines could power the radiative deexcitation causing the triplet line emission. That the forbidden doublet is also in emission implies continued radiative deexcitation before the normal collisional de-excitation (or, less likely, collisional re-excitation) occurs from this level of the permitted line. However, these phenomena are occurring at different velocities in the different Ca II lines in PTF 14jg, and thus the relation between the lines is unclear.

The observed ratio of $\sim 9$ in the emission peaks of $\mathrm{Ca}$ II $8542 \AA$ to [Ca II] $7291 \AA$ (Figure 12, showing various epochs) corresponds to an electron density of several times $10^{8} \mathrm{~cm}^{-3}$, independent of temperature, according to Figure 8 from Nisini et al. (2005). Figure 2 from Ferland \& Persson (1989) suggests a few times $10^{9} \mathrm{~cm}^{-3}$. The implied high density suggests that collisions are more important than radiation in forming the emission lines, but again, the very different morphologies of the permitted versus forbidden lines may invalidate the calculation. The low-velocity gas-where the forbidden (and other neutral atomic) emission arises-may be at low density, whereas the higher-velocity gas may have higher density. This would be consistent with a wind that is accelerated within a dense disk (forming the broad, permitted triplet emission) and launched into a less dense circumstellar medium, where it decelerates (forming the narrow forbidden doublet emission).

\subsection{Progenitor}

The pre-outburst nature of PTF 14jg is poorly constrained. Considering the immediate pre-outburst brightness of $R_{\mathrm{PTF}} \approx 21.5$ and the plausible range of spectral types and extinctions that are consistent with the limited SED information, the luminosity estimate is $\sim 0.1-0.3 L_{\odot}$ for the $2 \mathrm{kpc}$ distance. These values would be typical for low-mass $\left(<0.5 M_{\odot}\right)$ young pre-main-sequence stars.

Using the Baraffe et al. (1998) evolutionary models implemented within TADA (da Rio et al. 2012), the faint pre-outburst PTF and Spitzer/IRAC photometry would correspond to just $\sim 0.05,0.2$, and $0.5 M_{\odot}$ objects for ages of 1,10 , and 100 Myr. Adopting the source extinction estimate of $A_{V}=4.75 \mathrm{mag}$ has little effect on these mass numbers. Even if it is an older field object in the Perseus arm, and not a young source associated with $W 3 / W 4 / W 5$, the PTF $14 \mathrm{jg}$ progenitor is implied to have subsolar mass, given its brightness.

Using another line of argument, if the $\approx 500 \mathrm{~km} \mathrm{~s}^{-1}$ that is observed as the maximum terminal velocity in the various (accretion-driven) wind-dominated lines can be interpreted as the escape speed, this would correspond to $3.0,0.8$, and $0.5 M_{\odot}$. stars for the radii that are implied at ages of $\approx 1,3$, and $10 \mathrm{Myr}$.

\section{PTF 14jg Characteristics in the Context of Other Possible Interpretations}

Besides the FU Ori event interpretation discussed above, what other type of large-amplitude photometric rise would have characteristics like what we observe for PTF 14jg? The salient features of the outburst are as follows.

1. A progenitor of $\sim 0.1-0.3 L_{\odot}$ and, if a pre-main-sequence star, approximately $0.2-0.5 M_{\odot}$.

2. Source brightening by 6-7 mag over a time period of a few months.

3. Exponential decline from light-curve peak with an $e$-folding time of $\sim 111$ days, reaching a plateau $\sim 3$ mag below peak and $\sim 3.5-4$ mag above quiescence.

4. Initially colorless fade in the optical, though exhibiting a later reddening trend in optical and near-infrared colors, and possible blueing in [3.5]-[4.5] color.

5. Peak luminosity of $100-130 L_{\odot}$

6. Ultraviolet, near-infrared, and mid-infrared excess.

7. A low-gravity (supergiant), composite spectrum with a systematically changing temperature going from bluer optical wavelengths, where it best matches an early-A spectral type (though lacking strong hydrogen lines), to 
redder optical wavelengths, where it is best matched to a $\mathrm{G}$ or even $\mathrm{K} 0$ spectral type.

8. Line broadening of $\sim 150 \mathrm{~km} \mathrm{~s}^{-1}$ in the optical absorption spectrum.

9. Only very weak and blueshifted absorption in $\mathrm{HI}$ and He I lines.

10. Strong and highly blueshifted $\left(500 \mathrm{~km} \mathrm{~s}^{-1}\right.$ terminal velocity) absorption in the $\mathrm{Ca}$ II $\mathrm{H} \& \mathrm{~K}$ resonance lines, $\mathrm{NaD}$, and $\mathrm{O}$ I, which are often seen in winds, as well as less typical species, such as low-excitation Fe II and higher-excitation $\mathrm{Si}$ II and $\mathrm{Mg}$ II.

11. Moderate $\mathrm{Ca}$ II triplet and forbidden [Ca II] emission with early-time kinematic structure.

12. Narrow ( $\left.\sim 25-30 \mathrm{~km} \mathrm{~s}^{-1}\right)$ and symmetric neutral species atomic emission from, e.g., Fe I, Mg I, and $\mathrm{Ca}$ I that was observed initially in the optical and near-infrared for at least 6 weeks post-peak but disappeared by about 6 months after the light-curve peak.

13. An outburst lifetime lasting at least $5 \mathrm{yr}$.

We have considered a wide range of explanations that are summarized in Table 7. Most known categories of largeamplitude instabilities or explosive events can be ruled out by the long rise time and relatively slow decay time of PTF 14jg, along with its only moderate outburst luminosity. Other explanations are ruled out by the lack of a dust formation phase and/or late-time emission spectrum phase.

We also note that over the several years of high-dispersion follow-up data, there is no evidence for radial velocity variation in the emerging absorption spectrum. This eliminates a certain parameter space of binarity and thus close binary interaction as an explanation for the photometric outburst.

\section{Discussion}

Our conclusion from the previous two sections is that PTF 14jg bears some resemblance to FU Ori stars, albeit with many unusual characteristics, and that all other possible explanations for the large-amplitude photometric increase and warm spectrum encounter insurmountable difficulties. The evidence for preexisting circumstellar dust, plus the composite absorption spectrum, the broad absorption lines, and the strong wind-all sustained several years post-peak-suggests an accretion-driven burst accompanied by an accelerated wind. We thus consider it an acceptable hypothesis that PTF $14 \mathrm{jg}$ is just an unusually hot FU Ori disk.

In classical viscous accretion disk theory (Lynden-Bell \& Pringle 1974), the maximum temperature of the disk occurs at $\approx 1.4 R_{*}$ and has a value of $0.49\left(3 G \dot{M} M_{*} / 8 \pi \sigma R_{*}^{3}\right)^{1 / 4}$, with temperature falling off as $r^{-3 / 4}$. A high disk temperature, such as we observe, thus requires one or more of the following: high mass accretion rate, high stellar mass, or small stellar radius compared to a canonical FU Ori disk. As shown in Figure 18, a mass above $5 M_{\odot}$, radius closer to $1 R_{\odot}$, or very high accretion rate above $10^{-4} M_{\odot} \mathrm{yr}^{-1}$ (with the other parameters fixed) would be required in order to raise the inner disk temperature above $10,000 \mathrm{~K}$.

Guided by the implied low luminosity in the pre-outburst phase, we can consider a low-mass star in the range $0.1-0.5 M_{\odot}$ with a $0.8-1.2 R_{\odot}$ radius that would give the observed disk temperature. This object (magenta line in the figure) would have an age of a few Myr instead of the $<1$ Myr age of the fiducial case. It would thus be a late-stage FU Ori event. We note that this argument is consistent with the inferred source luminosity. Rewriting the above equation in terms of accretion luminosity, we find $T_{\max }=3700\left(L_{\mathrm{disk}} / L_{\odot}\right)^{1 / 4}$ $(R / R \odot)^{-1 / 2}$, and for $T_{\max }=12,000 \mathrm{~K}$ and $L_{\text {disk }}=L=$ $114 L_{\odot}$, that $R=0.95 R_{\odot}$ is implied.

If alternate merge-burst or other explosive scenarios are correct, then there would have been rapid evolution (on roughly a monthlong timescale) in the light-curve decline and the spectrum from early to late type as an expanding shell cooled. Instead, the light curve of PTF 14jg was only slowly declining, and there was no evolution in the spectrum over several months.

A faster photometric decay was experienced by PTF14jg than by most other known FU Ori-type bursts. However, 4 yr post-outburst, it was still 3.5-4 mag above quiescence. Its optical rise amplitude of $6.7 \mathrm{mag}$ was also larger than most FU Ori bursts. And the initial spectrum was hotter. Despite the differences relative to canonical FU Ori stars, if PTF 14jg is indeed a member of this class, it is one of $<10$ young stars to have been caught in the act, that is, to have had its outburst phase fully captured and well documented (better than almost all, in fact). Continued monitoring of the light curve and spectral evolution will reveal its true nature.

Among young starbursts and outbursts, the popular FU Ori and EX Lup classes differ in their amplitudes, durations, and duty cycles, as well as in their physical interpretation. The former are large-scale disk instabilities, while the latter smallerscale events have a probable origin in the magnetospheric region. However, other young star outburst types are beginning to be characterized in the literature, and the landscape of burst amplitude and timescale is not yet fully appreciated. These new object types include those with EX Lup-type amplitudes but even shorter-duration (a few months) outbursts, such as ASASSN13db (5.4 mag, < 150 days; Holoien et al. 2014), PTF 15afq (3.5 mag, 200 days; Miller et al. 2015), V899 Mon (3 mag, $>10$ yr; Ninan et al. 2015), and ASAS-15qi (3.5 mag, 200 days; Herczeg et al. 2016), and the even lower-amplitude and more frequent (few days to a week) bursts associated with accreting stars as studied by Cody et al. (2017) in Upper Sco and Stauffer et al. $(2014,2016)$ in NGC 2264. PTF 14jg may be an example of similar diversity among the larger-amplitude FU Ori-type events.

The phase space of the amplitude timescale for young star outbursts is still being mapped out. Time-domain surveys are now producing quality light curves of young stars with roughly monthlong (e.g., the YSOVAR surveys with Spitzer, the K2 mission, the VVV survey) to years-long (e.g., ASAS, PTF, Pan-STARRS, ZTF, ATLAS) duration. Our understanding of the range of burst and outburst behavior exhibited by young stars will thus continue to grow. Worth remembering is the reminder by Herczeg et al. (2016): "measuring the duration of an outburst usually requires impatient people to wait."

\section{Conclusions}

We have presented the outburst of PTF 14jg near the W4 H II region, documenting the early history of its photometric and spectroscopic behavior.

The rise time and amplitude of the PTF 14jg outburst are roughly consistent with an FU Orionis classification, though it is at an extreme in both parameters relative to other members of 
Table 7

PTF14jg Hypothesis Matrix

\begin{tabular}{|c|c|c|c|c|c|c|c|c|c|c|c|c|c|c|c|}
\hline \multirow[t]{2}{*}{ Phenomenon } & \multirow[t]{2}{*}{ Examples } & \multicolumn{14}{|c|}{ Is the Observed Property in PTF 14jg Consistent with the Category? } \\
\hline & & $\begin{array}{c}\text { Pre- } \\
\text { Burst } \\
\text { Variab. }\end{array}$ & $\begin{array}{l}\text { Rise } \\
\text { Time }\end{array}$ & $\Delta \mathrm{mag}$ & $\begin{array}{l}\text { Blue } \\
+ \text { Hot } \\
\text { Burst } \\
\end{array}$ & $\begin{array}{l}\text { Colorless } \\
\text { Fade }\end{array}$ & $\begin{array}{c}\text { Decay } \\
\text { Time }\end{array}$ & $\begin{array}{l}\text { 2nd } \\
\text { Peak }\end{array}$ & $\begin{array}{l}\text { Low } \\
\text { Lum. }\end{array}$ & $\begin{array}{l}\text { Infrared } \\
\text { Excess }\end{array}$ & $\begin{array}{l}\text { Wind } \\
\text { Speed }\end{array}$ & $\begin{array}{l}\text { Weak } \\
\mathrm{H} \alpha \\
\text { abs. }\end{array}$ & $\begin{array}{l}\text { Low eV } \\
\text { Narrow } \\
\text { emis. }\end{array}$ & $\begin{array}{c}\text { High eV } \\
\text { Broad } \\
\text { abs. }\end{array}$ & $\begin{array}{l}\text { Spec. } \\
\text { Evol. }\end{array}$ \\
\hline \multicolumn{16}{|c|}{ Accretion-Related Events } \\
\hline $\begin{array}{l}\text { Young star outburst } \\
\text { (FU Ori event) }\end{array}$ & $\begin{array}{l}\text { V1057 Cyg } \\
\text { PTF 10qpf }\end{array}$ & $\checkmark$ & $\checkmark$ & $x$ & $\times$ & $\checkmark$ & $\times$ & $\checkmark$ & $\checkmark$ & $\checkmark$ & $\checkmark$ & $\times$ & $x$ & $\checkmark$ & $\checkmark$ \\
\hline Symbiotic binary nova & $\begin{array}{l}\text { V694 Mon } \\
\text { FG Ser }\end{array}$ & & & $\checkmark$ & $\checkmark$ & & $\checkmark$ & & $\times$ & $\checkmark$ & $\checkmark$ & $\times$ & & $\times$ & $\times$ \\
\hline Classical nova & $\begin{array}{c}\text { RR Pic } \\
\text { V4739 Sgr } \\
\text { V3890 Sgr }\end{array}$ & $\times$ & $\times$ & $\checkmark$ & $\checkmark$ & & $\times$ & $\checkmark$ & $\times$ & $\checkmark$ & & $\times$ & & & $\times$ \\
\hline Slow nova & $\begin{array}{c}\text { T Pyx } \\
\text { V2540 Oph } \\
\text { V5558 Sgr }\end{array}$ & & $x$ & $\checkmark$ & $\checkmark$ & $\times$ & & $\checkmark$ & $x$ & & & $x$ & & & $\times$ \\
\hline Pre-cataclysmic nova & $\begin{array}{l}\text { U Gem } \\
\text { SS Cyg } \\
\text { Z Cam }\end{array}$ & & $x$ & $\checkmark$ & $x$ & $\times$ & $x$ & & $x$ & & & $\times$ & & & $\times$ \\
\hline \multicolumn{16}{|c|}{ Nuclear-Burning Instabilities } \\
\hline Helium-flash instability & V445 Pup & & & & $\checkmark$ & & & & $\times$ & $\checkmark$ & & $\checkmark$ & $\checkmark$ & $\times$ & $\times$ \\
\hline $\begin{array}{l}\text { Final helium flash } \\
\text { (“Born again" star) }\end{array}$ & $\begin{array}{c}\text { FG Sgr } \\
\text { V4334 Sgr } \\
\text { V605 Aql }\end{array}$ & $x$ & $\checkmark$ & $\checkmark$ & & $x$ & $x$ & & & & & $\checkmark$ & & & $\times$ \\
\hline \multicolumn{16}{|c|}{ Massive Star Behavior } \\
\hline $\begin{array}{l}\text { Red supergiant } \\
\text { LPV pulsator }\end{array}$ & $\begin{array}{c}\text { Mira } \\
\text { V566 Cas }\end{array}$ & $x$ & $\checkmark$ & $\checkmark$ & $x$ & & $\checkmark$ & $x$ & $x$ & $\checkmark$ & & $x$ & & & $x$ \\
\hline Yellow hypergiant burst & $\rho$ Cas & & & & $\checkmark$ & & & & $x$ & & $\times$ & & & & $\times$ \\
\hline $\begin{array}{l}\text { Blue supergiant } \\
\text { LBV outburst }\end{array}$ & $\begin{array}{c}\eta \text { Car } \\
\text { P Cyg } \\
\text { AG Car }\end{array}$ & $\checkmark$ & $x$ & $\checkmark$ & $x$ & & $\checkmark$ & & $x$ & & $x$ & $x$ & $x$ & $x$ & $x$ \\
\hline Supernova imposter & UGC 2773-OT & $x$ & $\checkmark$ & $x$ & $\checkmark$ & & & & $x$ & & $x$ & $x$ & $x$ & $x$ & $\times$ \\
\hline \multicolumn{16}{|c|}{ Merger Events } \\
\hline Star-star merger & $\begin{array}{l}\text { V1309 Sco } \\
\text { V838 Mon } \\
\text { V4332 Sgr }\end{array}$ & $\checkmark$ & $x$ & $x$ & $x$ & & $x$ & $x$ & $x$ & $\checkmark$ & $x$ & $x$ & $x$ & $x$ & $x$ \\
\hline Star-planet merger & Theoretical & & $\checkmark$ & & $x$ & & $x$ & & $x$ & & & & & & $x$ \\
\hline
\end{tabular}

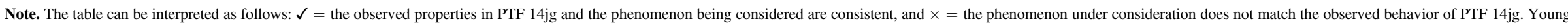
star outbursts have the most properties in common with PTF 14jg. Almost all of the other hypotheses fail to replicate the low luminosity or weak absorption spectrum observed in PTF 14jg. 


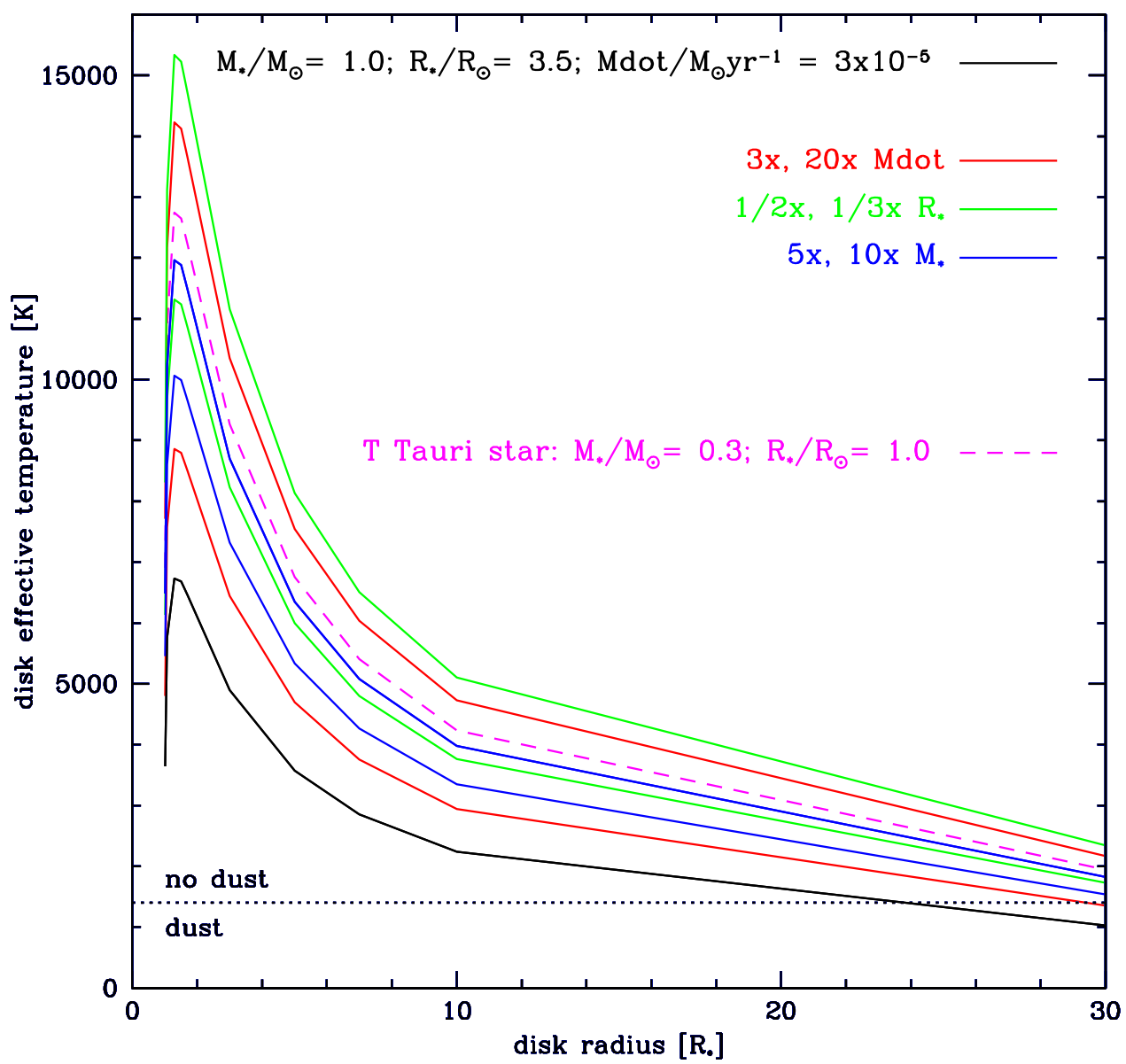

Figure 18. Classical accretion disk models showing the plausible parameters that could produce a disk with maximum temperature in the range required by the broadlined absorption spectrum of PTF 14jg. A fiducial FU Ori disk that reaches a maximum temperature of close to $7000 \mathrm{~K}$ is shown in black. Red lines indicate increased accretion rate, green lines indicate decreased stellar radius, and blue lines indicate increased stellar mass. The dashed magenta line shows the stellar parameters for a young pre-main-sequence star having an inferred pre-outburst luminosity of $0.1 L_{\odot}$, which, in addition to the implied lower mass, has a smaller radius (i.e., older premain-sequence age of 2-3 Myr) relative to the fiducial solar-mass case, in order to achieve the required higher disk temperature. Other combinations are possible.

the class. The characteristic change in spectral type with wavelength that is a hallmark of the class is present, as is Li I absorption. However, the spectral signatures are overall hotter in the early stages, both in the rest velocity (disk) spectrum and the outflowing (wind) spectrum, compared to other FU Ori stars. As shown in Figure 18, the required temperatures can be generated by a disk around a $0.3 M_{\odot}, 1.0 R_{\odot}$ star having an accretion rate of $3 \times 10^{-5} M_{\odot} \mathrm{yr}^{-1}$. This would be a $1-3 \mathrm{Myr}$ old progenitor rather than the more typically considered $<1$ Myr old FU Ori source.

Our classification of PTF 14jg as a member of the FU Ori class is tentative. We also considered a large body of alternate explanations for our suite of observations. However, we find no clearly better, or even equally good, alternative to the FU Ori outburst interpretation. Continued spectral study as the source cools from its peak brightness, especially at infrared wavelengths, should better illuminate its nature.

We have made extensive use of SIMBAD and ADS online resources in sleuthing for possible analogs of PTF 14jg. We also spent a considerable amount of quality time with the NIST atomic database in attemping line identification in the HIRES data, and we consulted the MILES population synthesis spectral database when searching for spectral templates. We are grateful to Cathie Clarke and Giuseppe Lodato for locating electronic copies of photometric data on the "classical" FU Ori outbursts that were published in their 2005 paper and to Ariel Langer, who contributed to our analysis of these light curves. We thank Mark Heyer for locating CO channel maps from his 1998 paper. We benefited from allocations by Tom Soifer as director of the Spitzer Space Telescope and Lee Mundy as director of CARMA of small amounts of DDT to obtain the observations reported here. We thank the spectroscopic observers listed in Table 6 who are not otherwise acknowledged with authorship, especially Yi Cao, Sumin Tang, Jacob Jencson, and Anna Ho. D.P.K.B. thanks N. M. Ashok and V. Venkataraman for help with some of the Mount Abu observations. The research at PRL is supported by the Department of Space, Government of India. We thank the staff members at the various observational facilities used and the numerous instrument builders. The Intermediate Palomar Transient Factory project is a scientific collaboration among the California Institute of Technology, Los Alamos National Laboratory, the University of Wisconsin-Milwaukee, the Oskar Klein Center, the Weizmann Institute of Science, the TANGO Program of the University System of Taiwan, and the Kavli Institute for the Physics and Mathematics of the 
Universe. Finally, L.A.H. is grateful for the tolerance of the many young star and nova pundits on whom various versions of this story have been tried out over the past several years. And we thank the referee for comments that provided a valuable opportunity for us to reexamine our presentation.

Facilities: PO:1.2m:PTF, PO:1.5m:GRBcam, MIRO:1.2m, Hale:DBSP, Hale:TSPEC, Keck:I:HIRES, Keck:I:LRIS, Keck: I:DEIMOS, Keck:I:MOSFIRE, APO:DIS, APO:TSPEC, 2MASS, Spitzer, WISE(NEOWISE), Swift:UVOT, Swift:XRT, CARMA, IRSA.

\section{ORCID iDs}

Adam A. Miller (1) https://orcid.org/0000-0001-9515-478X John M. Carpenter (i) https://orcid.org/0000-0003-2251-0602 Mansi M. Kasliwal (i) https://orcid.org/0000-0002-5619-4938 Howard Isaacson (1) https://orcid.org/0000-0002-0531-1073

Sumin Tang (i) https://orcid.org/0000-0002-6225-8918 Vishal Joshi (i) https://orcid.org/0000-0002-1457-4027 Roc M. Cutri (10) https://orcid.org/0000-0002-0077-2305

\section{References}

Abt, H. A., \& Morrell, N. I. 1995, ApJS, 99, 135

Anandarao, B., Richardson, E. H., Chakraborty, A., \& Epps, H. 2008, Proc. SPIE, 7014, 70142Y

Azevedo, R., Calvet, N., Hartmann, L., et al. 2006, A\&A, 456, 225

Banerjee, D. P. K., \& Ashok, N. M. 2012, BASI, 40, 243

Baraffe, I., Chabrier, G., Allard, F., \& Hauschildt, P. H. 1998, A\&A, 337, 403

Beckwith, S. V. W., Sargent, A. I., Chini, R. S., \& Guesten, R. 1990, AJ, 99, 924

Bertin, E., \& Arnouts, S. 1996, A\&AS, 117, 393

Breeveld, A. A., Curran, P. A., Hoversten, E. A., et al. 2010, MNRAS, 406, 1687

Carpenter, J. M., Heyer, M. H., \& Snell, R. L. 2000, ApJS, 130, 381

Cenko, S. B., Fox, D. B., Moon, D.-S., et al. 2006, PASP, 118, 1396

Churchwell, E., Babler, B. L., Meade, M. R., et al. 2009, PASP, 121, 213

Clarke, C., Lodato, G., Melnikov, S. Y., \& Ibrahimov, M. A. 2005, MNRAS, 361,942

Cody, A. M., Hillenbrand, L. A., David, T. J., et al. 2017, ApJ, 836, 41

Cushing, M. C., Rayner, J. T., \& Vacca, W. D. 2005, ApJ, 623, 1115

Cutri, R. M., Mainzer, A., Conrow, T., et al. 2012, yCat, 2311

Cutri, R. M., Skrutskie, M. F., van Dyk, S., et al. 2003, yCat, 2246

da Rio, N., Robberto, M., Hillenbrand, L. A., Henning, T., \& Stassun, K. G. 2012, ApJ, 748, 14

Ferland, G. J., \& Persson, S. E. 1989, ApJ, 347, 656

Georgelin, Y. P., \& Georgelin, Y. M. 1970, A\&A, 6, 349

Gramajo, L. V., Rodón, J. A., \& Gómez, M. 2014, AJ, 147, 140

Grinin, V. P., Kozlova, O. V., Natta, A., et al. 2001, A\&A, 379, 482

Gullbring, E., Hartmann, L., Briceno, C., \& Calvet, N. 1998, ApJ, 492, 323
Hartigan, P., Edwards, S., \& Pierson, R. 2004, ApJ, 609, 261

Hartmann, L., \& Kenyon, S. J. 1996, ARA\&A, 34, 207

Herbig, G. H. 1977, ApJ, 217, 693

Herbig, G. H. 2009, AJ, 138, 448

Herczeg, G. J., Dong, S., Shappee, B. J., et al. 2016, ApJ, 831, 133

Heyer, M. H., Brunt, C., Snell, R. L., et al. 1998, ApJS, 115, 241

Heyer, M. H., Carpenter, J. M., \& Snell, R. L. 2001, ApJ, 551, 852

Hillenbrand, L. A., Contreras Peña, C., Morrell, S., et al. 2018, ApJ, 869, 146

Hinkley, S., Hillenbrand, L., Oppenheimer, B. R., et al. 2013, ApJL, 763, L9

Holoien, T. W.-S., Prieto, J. L., Stanek, K. Z., et al. 2014, ApJL, 785, L35

Hora, J., Adams, J., Allen, L., et al. 2007, sptz prop, 40184

Königl, A., Romanova, M. M., \& Lovelace, R. V. E. 2011, MNRAS, 416, 757

Kraus, S., Caratti o Garatti, A., Garcia-Lopez, R., et al. 2016, MNRAS, 462, L61

Kudritzki, R. P., Puls, J., Lennon, D. J., et al. 1999, A\&A, 350, 970

Kulkarni, S. R. 2013, ATel, 4807

Kurosawa, R., \& Romanova, M. M. 2012, MNRAS, 426, 2901

Lagrois, D., \& Joncas, G. 2009, ApJ, 691, 1109

Laher, R. R., Surace, J., Grillmair, C. J., et al. 2014, PASP, 126, 674

Law, N. M., Kulkarni, S. R., Dekany, R. G., et al. 2009, PASP, 121, 1395

Lefever, K., Puls, J., Morel, T., et al. 2010, A\&A, 515, A74

Leggett, S. K., Currie, M. J., Varricatt, W. P., et al. 2006, MNRAS, 373, 781

Lynden-Bell, D., \& Pringle, J. E. 1974, MNRAS, 168, 603

Mainzer, A., Bauer, J., Cutri, R. M., et al. 2014, ApJ, 792, 30

Masci, F. J., Laher, R. R., Rebbapragada, U. D., et al. 2017, PASP, 129, 014002

Megeath, S. T., Townsley, L. K., Oey, M. S., \& Tieftrunk, A. R. 2008, in Handbook of Star-forming Regions Vol. I, ed. B. Reipurth (San Francisco, CA: ASP), 264

Merrill, P. W. 1943, PASP, 55, 242

Miller, A. A., Hillenbrand, L. A., Bilgi, P., et al. 2015, ATel, 7428

Miller, A. A., Hillenbrand, L. A., Covey, K. R., et al. 2011, ApJ, 730, 80

Ninan, J. P., Ojha, D. K., Baug, T., et al. 2015, ApJ, 815, 4

Nisini, B., Bacciotti, F., Giannini, T., et al. 2005, A\&A, 441, 159

Ofek, E. O., Laher, R., Surace, J., et al. 2012, PASP, 124, 854

Rei, A. C. S., Petrov, P. P., \& Gameiro, J. F. 2018, A\&A, 610, A40

Reipurth, B., \& Aspin, C. 2010, in Proc. VAOA Conf., Evolution of Cosmic Objects through their Physical Activity, ed. H. A. Harutyunian, A. M. Mickaelian, \& Y. Terzian (Yerevan: NAS RA), 19

Roming, P. W. A., Kennedy, T. E., Mason, K. O., et al. 2005, SSRv, 120, 95

Semkov, E. H., Peneva, S. P., \& Ibryamov, S. I. 2017, BlgAJ, 26, 57

Semkov, E. H., Peneva, S. P., Munari, U., Milani, A., \& Valisa, P. 2010, A\&A, 523, LL3

Silva, D. R., \& Cornell, M. E. 1992, ApJS, 81, 865

Soubiran, C., Bienaymé, O., Mishenina, T. V., \& Kovtyukh, V. V. 2008, A\&A, 480,91

Stauffer, J., Cody, A. M., Baglin, A., et al. 2014, AJ, 147, 83

Stauffer, J., Cody, A. M., Rebull, L., et al. 2016, AJ, 151, 60

Strope, R. J., Schaefer, B. E., \& Henden, A. A. 2010, AJ, 140, 34

Terebey, S., Fich, M., Taylor, R., Cao, Y., \& Hancock, T. 2003, ApJ, 590, 906

Vazdekis, A., Cenarro, A. J., Gorgas, J., Cardiel, N., \& Peletier, R. F. 2003, MNRAS, 340, 1317

Verdugo, E., Talavera, A., \& Gómez de Castro, A. I. 1999, A\&A, 346, 819

Xu, Y., Reid, M. J., Menten, K. M., \& Zheng, X. W. 2006, ApJS, 166, 526

York, D. G., Adelman, J., Anderson, J. E., Jr., et al. 2000, AJ, 120, 1579 